Linköping Studies in Science and Technology.

Dissertations No. 1724

\title{
Contributions to High-Dimensional Analysis under Kolmogorov Condition
}

\author{
Jolanta Maria Pielaszkiewicz
}

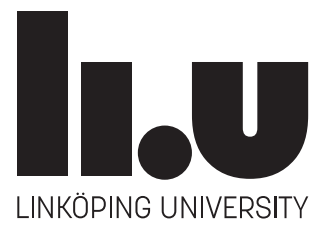

Department of Mathematics, Division of Mathematical Statistics Linköping University, SE-581 83 Linköping, Sweden

Linköping 2015 
Linköping Studies in Science and Technology. Dissertations No. 1724 Contributions to High-Dimensional Analysis

Copyright (C) Jolanta Maria Pielaszkiewicz, 2015

Division of Mathematical Statistics

Department of Mathematics

Linköping University

SE-581 83, Linköping, Sweden

jolanta.pielaszkiewicz@liu.se

www.liu.se/mai/ms

Typeset by the author in $\mathrm{HT}_{\mathrm{E}} \mathrm{X} 2 \mathrm{e}$ documentation system.

ISSN 0345-7524

ISBN 978-91-7685-899-8

Printed by LiU-Tryck, Linköping, Sweden 2015 


\section{Abstract}

This thesis is about high-dimensional problems considered under the so-called Kolmogorov condition. Hence, we consider research questions related to random matrices with $p$ rows (corresponding to the parameters) and $n$ columns (corresponding to the sample size), where $p>n$, assuming that the ratio $\frac{p}{n}$ converges when the number of parameters and the sample size increase.

We focus on the eigenvalue distribution of the considered matrices, since it is a well-known information-carrying object. The spectral distribution with compact support is fully characterized by its moments, i.e., by the normalized expectation of the trace of powers of the matrices. Moreover, such an expectation can be seen as a free moment in the non-commutative space of random matrices of size $p \times p$ equipped with the functional $\frac{1}{p} \mathbb{E}[\operatorname{Tr}\{\cdot\}]$. Here, the connections with free probability theory arise. In the relation to that field we investigate the closed form of the asymptotic spectral distribution for the sum of the quadratic forms. Moreover, we put a free cumulant-moment relation formula that is based on the summation over partitions of the number. This formula is an alternative to the free cumulant-moment relation given through non-crossing partitions of the set.

Furthermore, we investigate the normalized $\mathbb{E}\left[\prod_{i=1}^{k} \operatorname{Tr}\left\{W^{m_{i}}\right\}\right]$ and derive, using the differentiation with respect to some symmetric matrix, a recursive formula for that expectation. That allows us to re-establish moments of the MarčenkoPastur distribution, and hence the recursive relation for the Catalan numbers.

In this thesis we also prove that the $\prod_{i=1}^{k} \operatorname{Tr}\left\{W^{m_{i}}\right\}$, where $W \sim \mathcal{W}_{p}\left(I_{p}, n\right)$, is a consistent estimator of the $\mathbb{E}\left[\prod_{i=1}^{k} \operatorname{Tr}\left\{W^{m_{i}}\right\}\right]$. We consider

$$
Y_{t}=\sqrt{n p}\left(\frac{1}{p} \operatorname{Tr}\left\{\left(\frac{1}{n} W\right)^{t}\right\}-m_{1}^{(t)}(n, p)\right),
$$

where $m_{1}^{(t)}(n, p)=\mathbb{E}\left[\frac{1}{p} \operatorname{Tr}\left\{\left(\frac{1}{n} W\right)^{t}\right\}\right]$, which is proven to be normally distributed. Moreover, we propose, based on these random variables, a test for the identity of the covariance matrix using a goodness-of-fit approach. The test performs very well regarding the power of the test compared to some presented alternatives for both the high-dimensional data $(p>n)$ and the multivariate data $(p \leq n)$. 



\section{Populärvetenskaplig sammanfattning}

I många tillämpningar förekommer slumpmatriser, det vill säga matriser vars element följer någon stokastisk fördelning. Det är ofta intressant att känna till hur egenvärdena för dessa slumpmässiga matriser uppför sig, det vill säga beräkna fördelningen för egenvärdena, den så kallade spektralfördelningen. Egenvärdena är informationsbärande objekt, då de ger information om till exempel stabilitet och inversen av den slumpmässiga matrisen genom det minsta egenvärdet.

Telekommunikation och teoretisk fysik är två områden där det är intressant att studera slumpmatriser, matrisernas egenvärden och fördelningen för dessa egenvärden. Speciellt gäller det för stora slumpmatriser där man då är intresserad av den asymptotiska spektralfördelningen. Ett exempel kan vara en kanalmatris $X$ för ett flerdimensionellt kommunikationssystem, där fördelningen för egenvärdena för matrisen $X X^{*}$ bestämmer kanalkapaciteten och uppnåeliga överföringshastigheter.

Spektralfördelningen har studerats ingående inom teorin för slumpmässiga matriser. I denna avhandling kombinerar vi resultaten från teorin för slumpmatriser tillsammans med idén om fritt oberoende som diskuterades först av Voiculescu (1985). Högdimensionella problem som kan komma ifråga under så kallade Kolmogorov villkor diskuteras. Vi betraktar alltså forskningsfrågor som rör slumpmatriser med $p$ rader (motsvarande antalet parametrar) och $n$ kolumner (motsvarande stickprovets storlek), där $p>n$, och förutsatt att kvoten $\frac{p}{n}$ förblir konstant då antalet parametrar och stickprovsstorleken ökar. 



\section{Acknowledgments}

There are many people who have influenced writing of this thesis, one way or the other, and I would like to thank them all.

Foremost, I would like to express my gratitude towards Professor Dietrich von Rosen for all the advice, support and guidance I have been lucky to get during my work at LiU. I thank him for all the insightful discussions that always gave me additional energy for conducting research. I also appreciate all the valuable comments on the various drafts of the thesis.

I thank my second supervisor Dr Martin Singull for discussions, "open door" attitude and all the help I got from him. We have been cooperating in so many ways and in each of them his meritorious help has been greatly appreciated.

I am grateful to both of my supervisors for the friendly atmosphere they created, for encouraging me and supporting all the different kinds of academic activities in which I have participated.

I have a strong belief that I would not be in the place I am right now without Professor Torbjörn Larsson. Thank you.

I am grateful to my colleagues at the Department of Mathematics LiU, especially those with whom I had the opportunity to cooperate or study. I thank Dr John Noble and Prof. Stefan Rauch for a number of good advice. I appreciate administrative help of Theresia, Monika, Eija, Elaine and Karin. I thank also Ingegerd for her help. Here, I cannot go without mentioning my colleagues, current and former PhD students who have been a great source of friendship, especially Sonja, Alexandra, Samira, Anna, Nisse and Spartak, together with Innocent, Joseph, Markus, Arpan and Mikael.

I would like to thank Bengt Ove Turesson and Martin Singull for giving me the opportunity to conduct lectures within the SIDA project.

My thanks go to the organizers of LinStat'2012 and MatTriad'2015 for giving me the opportunity to present my research as an invited speaker on their conferences. Here, I would like to acknowledge Dr Miguel Fonseca from Nova University of Lisbon, Portugal for inviting me to the special section of the second conference. Moreover, I would like to express my gratitude to the organizers of the 2014 Program in "Random Matrix Theory", Institute of Advanced Studies, Princeton, USA for the learning and discussing opportunity. I thank for financial support of GRAPES under The Swedish Research Students Conference in Statistics, 2013 and of grant SFB 878 - Groups, Geometry and Actions under Masterclass on Free Probability and Operator Algebras, Muenster, 2013.

Finally, I want to acknowledge my family for their love and faith in me. Thank you mam, dad, Tomek, Marysia, Pawel, Julek, Olek, Alicja, Serge and my dear Krzysiek. I thank my friends, who believed in me, from all around the world. 



\section{List of Papers}

The following papers are included in this thesis and will be referred to by their roman numerals.

I. J. Pielaszkiewicz and M. Singull, Closed form of the asymptotic spectral distribution of random matrices using free independence, Linköping University Electronic Press, Technical Report LiTH-MAT-R-2015/12-SE (2015).

II. J. Pielaszkiewicz, D. von Rosen and M. Singull, Cumulant-moment relation in free probability theory, Acta et Commentationes Universitatis Tartuensis de Mathematica 18(2) (2014) pp. 265-278.

III. J. Pielaszkiewicz, D. von Rosen and M. Singull, On $\mathbb{E}\left[\prod_{i=0}^{k} \operatorname{Tr}\left\{W^{m_{i}}\right\}\right]$, where $W \sim \mathcal{W}_{p}(I, n)$. Accepted for publication in Communications in Statistics - Theory and Methods (2015a).

IV. J. Pielaszkiewicz, D. von Rosen and M. Singull, On $p / n$-asymptotics applied to traces of 1 st and 2nd order powers of Wishart matrices with application to goodness-of-fit testing. Unpublished manuscript (2015b).

In all aforementioned works I have contributed by doing all detailed calculations, deriving the results and writing the papers. Since the considered research problems, and hence papers, were the product of multiple author discussions, I would not like to claim that I am the only author of the ideas presented in the papers, apart from Paper IV where I stated the considered research question. All the connections to free probability, such as for example re-deriving the moments of the Marčenko-Pastur law, are my own work. Moreover, I have done all the coding and simulations. 



\section{Awards for conference presentations}

Material of the thesis has been presented in a number of conferences. On two occasions presentations have been awarded prizes for the best $\mathrm{PhD}$ student presentation.

I. 2nd Prize in Young Scientists Awards - LinStat'2012 (The International Conference on Trends and Perspectives in Linear Statistical Inference)

16-20 July 2012, Bedlewo, Poland

II. Prize in Young Scientists Awards - MatTriad'2015 (Conference on Matrix Analysis and its Applications)

7-11 September 2015, Coimbra, Portugal 



\section{Contents}

Abstract i

Populärvetenskaplig sammanfattning iii

Acknowledgments $\quad$ v

List of Papers vii

Awards for conference presentations ix

1 Introduction 1

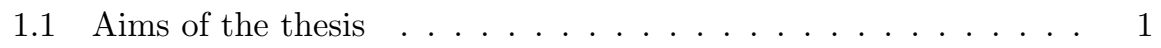

1.2 Outline of the thesis $\ldots \ldots \ldots \ldots \ldots$

2 Free probability theory 5

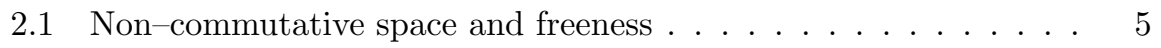

2.1 .1 Freeness . . . . . . . . . . . . . . . . 6

$2.1 .2 \quad$ Space $\left(R M_{p}(\mathbb{C}), \tau\right) \ldots \ldots \ldots \ldots \ldots \ldots$

2.1 .3 Asymptotic freeness . . . . . . . . . . . . . . . 10

2.2 Combinatorial interpretation of freeness and free cumulants . . . 11

2.3 Free moment-cumulant formulas . . . . . . . . . . . . . . . 13

3 Stieltjes and R-transform 17

3.1 Stieltjes transform . . . . . . . . . . . . . . . . 18

3.1.1 Stieltjes transform approach for random matrices . . . . . 20

3.2 R-transform . . . . . . . . . . . . . . . . . . 21

3.3 Use of Stieltjes and R-transforms for deriving spectral distribution 25

4 Wishart matrices 31

4.1 Wishart distribution . . . . . . . . . . . . . . . 31

4.2 Properties of Wishart matrices . . . . . . . . . . . . . 33

4.3 On $\prod_{i=0}^{k} \operatorname{Tr}\left\{W^{m_{i}}\right\} \ldots \ldots \ldots \ldots \ldots \ldots \ldots$

4.3.1 Real Wishart matrix . . . . . . . . . . . . . . 35

4.3.2 Complex Wishart matrix _. . . . . . . . . . . 38

4.3.3 Comparison of results for complex and real Wishart matrices 38

4.3.4 Asymptotic distribution of $\frac{1}{p} \operatorname{Tr}\left\{\left(\frac{1}{n} X X^{\prime}\right)^{t}\right\} \ldots \ldots . .40$

4.4 Testing the identity of covariance matrices . . . . . . . . . 43 
5 Summary of papers, conclusions and further research $\quad 49$

5.1 Summary of papers . . . . . . . . . . . . . . . . . . 49

5.2 Conclusions .......................... 51

5.3 Further research .................... . . 52

$\begin{array}{ll}\text { References } & 55\end{array}$

\section{INCLUDED PAPERS}

I. Closed form of the asymptotic spectral distribution of random matrices using free independence . . . . . . . . . . 6 63

Appendix - Paper I . . . . . . . . . . . . . . . . . . . . . . . . 89

II. Cumulant-moment relation in free probability theory . . . . . . . . . 93

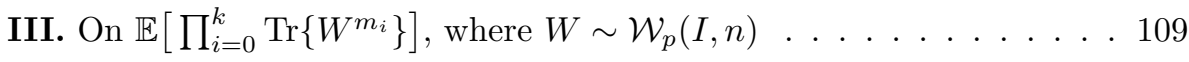
Appendix - Paper III . . . . . . . . . . . . . . . . . 129

IV. On $p / n$-asymptotics applied to traces of 1 st and 2 nd order powers of Wishart matrices with application to goodness-of-fit testing . 139 


\section{1}

\section{Introduction}

Nowadays a large amount of empirical problems generate high-dimensional data sets that are to be analyzed. One can mention genetics with DNA microarrays, physics, wireless communication or finance as examples of areas where assumptions about high dimension and large sample size apply. Since the amount of data increases in a number of different applications there is a strong interest to derive methods dealing with large-dimensional problems, such as problems related to large-size covariance matrices. Natural set-ups where the sample size $n$ is bigger than the number of parameters $p$ have been widely studied and one can refer here to classical books in multivariate analysis by Muirhead (1982) and Anderson (2003). In multivariate analysis one of the objects of great importance is the spectrum of covariance matrices that is utilized, for example, in principal components analysis. Many of the results given in the aforementioned books will not be true if the number of parameters were to exceed the sample size. Research problems under $p>n$ have been studied within the area of high-dimensional analysis, where, in particular, covariance estimation and regression have been investigated. Moreover, notice that in the case where $p>n$ the sample covariance matrix becomes singular. Hence, it cannot be inverted as demanded in a number of approaches. Problems with the assumption $p>n$ are, among others, considered by Pastur (1972) (studies on the spectral distribution of specific classes of random matrices), Mehta (1991) (a work of reference in random matrices) and Girko (1995) (studies on the distribution of eigenvalues).

\subsection{Aims of the thesis}

The aim of the thesis is to contribute to high-dimensional analysis through results based on classical moments, classical cumulants, free moments, free cumulants and particular form of so-called generalized moments. Since the $k$ th cumulant accumulates knowledge about moments up to the $k$ th degree, and vice versa, the analysis based on the moments is equivalent to the one done using cumulants. Thus, the thesis is about moments, cumulants and corresponding transforms.

In this thesis as well as in the appended papers, the matrix $X$ of size $p \times n$ represents a data matrix. Moreover, the asymptotic results are obtained under 
the Kolmogorov condition, i.e., $\frac{p}{n} \rightarrow c \in(0, \infty)$ while $n \rightarrow \infty$ and $p \rightarrow \infty$, see Paper I, III and IV. In other words we assume that both $p$ and $n$ increase with equal speed. Note that this condition is a special case of the G-condition, i.e., $\lim _{n \rightarrow} f(p, n)<\infty$ for some positive function $f(a, b)$ that decreases in $a$ and increases in $b$, where $f(a, b)=a b^{-1}$, see Girko (1990), Sinar (1991) and Girko (1995). Due to the development in technology, the assumption about high dimensionality of data becomes possible and the limiting results became realistic. The asymptotics under the Kolmogorov condition are also described as (n,p)-asymptotics (see, Ledoit and Wolf, 2002; Fujikoshi et al., 2011), increasing dimension asymptotic (Serdobolskii, 1999) or $p / n$-asymptotics (Kollo et al., 2011, and Paper III). Note that the formulas presented for $c \leq 1$ and $c>1$ consider multivariate and high-dimensional settings, respectively.

Keeping in mind the assumption about the speed of the increase in size of $n$ and $p$, we have investigated the possibility to obtain closed form expressions for the asymptotic spectral distribution function of a sum of matrix quadratic forms. This part of the research has been inspired by the paper of Girko and von Rosen (1994). Unlike the latter paper, we base our results on the properties and tools of free probability theory, such as asymptotic freeness (corresponding to independence in the classical probability space) and the R-transform (corresponds to the log of the Fourier transform). For now, free probability theory can be seen as the probability theory on a non-commutative space, such as the space of random matrices of fixed size. In the engineering examples, for instance in applications related to wireless communication, there is an interest in investigating the spectral measure of a sum of the quadratic forms. Moreover, the lack of the existence of a closed form expression for the spectral density is often pointed out, see e.g., Chen et al. (2012). For more information about free probability, see Chapter 2 in this thesis, Nica and Speicher (2006) and Hiai and Petz (2000).

The studies of free probability theory that were performed while working on closed form expressions for the asymptotic spectral distribution led us to the further investigation of the area. The combinatorial interpretation of free cumulants and their recursive definition based on the concept of non-crossing partitions are of particular interest to us, see Speicher (1994) and Nica and Speicher (2006). We develop however, a free cumulant-moment relation formula that omits the use of the concept of a non-crossing partition. One has to admit that the non-crossing partitions approach is very elegant and carries a lot of information, but at the same time it requires that users identify all non-crossing partitions over the set $\{1,2, \ldots, n\}$, which can be convenient to avoid.

The R-transform was studied in connection to the free cumulants as they carry the same information about the related compactly supported measure, see the original paper by Voiculescu (1991) and Chapter 3 in this thesis. Since the Stieltjes transform is related to both the moments and the R-transform, a relation between moments and cumulants can be derived as it was presented in Paper II. A number of cumulant-moment relation formulas were examined and 
led us to consider a research question about a formula for free moments in the following non-commutative space of random matrices, i.e., Wishart matrices within the framework of random matrices. The functional working on that space was chosen to be the expectation of the normalized trace of the matrix. Such a choice of functional relates our results to the spectral measure of the Wishart matrix. Moreover, as the Wishart matrix is symmetric (or Hermitian for complex Wishart matrices), the spectral measure is supported on the real line.

In Paper III we obtained $\mathbb{E}\left[\prod_{i=0}^{k} \operatorname{Tr}\left\{W^{m_{i}}\right\}\right]$ for a real Wishart matrix $W \sim$ $\mathcal{W}_{p}\left(I_{p}, n\right)$, where $I_{p}$ stands for the identity matrix of size $p$. Such an object is called the joint moment of the spectral distribution of $W$ (see, Nardo, 2014) or is a special case of the generalized moment of $W$ (see, Capitaine and Casalis, 2004, 2006).

The asymptotic version of the recursive formula for $\mathbb{E}\left[\prod_{i=0}^{k} \operatorname{Tr}\left\{W^{m_{i}}\right\}\right]$ implies that $\frac{1}{p} \operatorname{Tr}\left\{\left(\frac{1}{n} X X^{\prime}\right)^{t}\right\}$ has an asymptotic variance that equals zero for all $t$. Therefore, in Paper IV we investigated the marginal and joint distribution of $\left(Y_{1}, \ldots, Y_{k}\right)$, where

$$
Y_{t}=\sqrt{n p}\left(\frac{1}{p} \operatorname{Tr}\left\{\left(\frac{1}{n} X X^{\prime}\right)^{t}\right\}-\mathbb{E}\left[\frac{1}{p} \operatorname{Tr}\left\{\left(\frac{1}{n} X X^{\prime}\right)^{t}\right\}\right]\right) .
$$

Furthermore, in Paper IV we also developed an alternative to already existing tests for the identity of the covariance matrix based on a comparison between the expected and observed values of $Y_{t}$. Alternative tests have been developed by Ledoit and Wolf (2002), Srivastava (2005), Fisher et al. (2010), Fisher (2012) and several references therein.

This thesis contributes to high-dimensional analysis as it presents a new free cumulant-moment formula, develops an approach for stating a new recursive formula for generalized moments of a Wishart matrix $W_{p} \sim \mathcal{W}\left(I_{p}, n\right)$ in both finite and asymptotic regimes, investigates the possibility to obtain closed form solutions of the spectral density of the sum of asymptotic free quadratic forms, and finally proves normality of $Y_{t}$ given above, and proposes a new test for the identity of the covariance matrix. The testing method, in particular, is an interesting result in regards to its good performance for the high-dimensional data matrices.

\subsection{Outline of the thesis}

This thesis consists of two distinctive parts. The first part of the thesis consists of five chapters. In Chapter 1 the introduction to the thesis is presented by specifying aims and the outline of the work. In Chapters 2 to 4 , theoretical background on free probability, Stjeltjes and R-transform, Wishart matrices and testing independence are provided. In those chapters we briefly introduce the results necessary for further reading of the papers in the second part of the 
thesis. We also relate our contributions to other known results. Finally, the last chapter presents the summary of the included papers and a discussion regarding future research. The second part of the thesis consists of the four papers which have already been specified in the List of Papers on page vii. 


\section{Free probability theory}

The aim of this chapter is to provide a short introduction to free probability theory which will facilitate the comprehension of the papers in the second part of the thesis, especially Paper I and II. A number of definitions are presented in a general setting, as the results regarding the free moment-cumulant formula in Paper II are not limited to the space of random matrices on which we focus in the other papers. From Chapter 3 onwards, we will consider real Wishart matrices of size $p \times p$ as elements of the non-commutative space of all random matrices of the same size. Such a choice allows us to model random matrices asymptotically, under the Kolmogorov condition, and derive further results presented in Paper I, III and IV.

Free probability theory was settled by Voiculescu in the middle of the 1980's (Voiculescu, 1985), and together with the result published in Voiculescu (1991) regarding asymptotic freeness of random matrices, they established a new branch of theories and tools in Random matrix theory such as the R-transform. Freeness can also be studied using equivalent combinatorial definitions based on ideas of non-crossing partitions (see, Section 2.2). In the following chapter we introduce basic definitions and concepts of the theory using both random matrix theory and a combinatorial approach. These are introduced in the general set-up of a non-commutative probability space and specified in Section 2.1.2 under the algebra of random matrices.

\subsection{Non-commutative space and freeness}

In this section the goal is to present a concept of freeness in a non-commutative space, which is introduced according to, among others, Nica and Speicher (2006) and Voiculescu et al. (1992). Some properties for elements of a non-commutative space are also presented. For further reading, see Hiai and Petz (2000). 
Definition 2.1.1. A non-commutative probability space is a pair $(\mathcal{A}, \tau)$, where $\mathcal{A}$ is a unital algebra over the field of complex numbers $\mathbb{C}$ with identity element $1_{\mathcal{A}}$ and $\tau$ is a unital functional such that:

- $\tau: \mathcal{A} \rightarrow \mathbb{C}$ is linear,

- $\tau\left(1_{\mathcal{A}}\right)=1$.

Definition 2.1.2. The functional $\tau$ is called trace if $\tau(a b)=\tau(b a)$ for all $a, b \in \mathcal{A}$, where $\mathcal{A}$ is as in Definition 2.1.1.

Note that the word trace in this thesis has two meanings: trace as the name of the functional fulfilling $\tau(a b)=\tau(b a)$ for all $a, b \in \mathcal{A}$ and as the trace of a square matrix $A=\left(A_{i j}\right)$, i.e., $\operatorname{Tr} A=\sum_{i} A_{i i}$.

Definition 2.1.3. Let $\mathcal{A}$ in Definition 2.1.1 have a $*$-operation such that $*$ : $\mathcal{A} \rightarrow \mathcal{A},\left(a^{*}\right)^{*}=a$ and $(a b)^{*}=b^{*} a^{*}$ for all $a, b \in \mathcal{A}$ and let the functional $\tau$ satisfy

$$
\tau\left(a^{*} a\right) \geq 0 \quad \text { for all } a \in \mathcal{A} \text {. }
$$

Then, we call $\tau$ positive and $(\mathcal{A}, \tau) a *$-probability space.

Subsection 2.1.2 gives an example of a $*$-probability space with a positive tracial function, i.e., the space $\left(R M_{p}(\mathbb{C}), \tau\right)$ that is a space of $p \times p$ random matrices with entries being complex random variables with all moments finite and equipped with a functional such that $\tau(X):=\mathbb{E}\left(\operatorname{Tr}_{p}(X)\right)$, for all $X \in R M_{p}(\mathbb{C})$, where $\operatorname{Tr}_{p}=\frac{1}{p} \operatorname{Tr}$ is the standardized trace. In the next, we define random variables, moments and the distribution for elements in a non-commutative space.

\subsubsection{Freeness}

Freeness in a non-commutative probability space is the property that corresponds to independence in a classical probability space. In papers we often use freeness as it linearizes so-called free convolution. Furthermore, we later focus on independent Wishart matrices, that are proved to be asymptotically free objects.

Let us define the moment and $*$-distribution of $a \in \mathcal{A}$.

Definition 2.1.4.

a) The element $a \in \mathcal{A}$ is called a non-commutative random variable and $\tau\left(a^{j}\right)$ is its $j$ th moment for all $j \in \mathbb{N}$. The power of the element of the algebra, $a^{j}$, is well defined due to the fact that the algebra is closed under multiplication of elements, see Cameron (1998). 
b) Let $a \in \mathcal{A}$ be normal, i.e., $a a^{*}=a^{*} a$, where $\mathcal{A}$ denotes a *-probability space. If there exists a compactly supported probability measure $\mu$ on $\mathbb{C}$ such that

$$
\int z^{n} \bar{z}^{k} d \mu(z)=\tau\left(a^{n}\left(a^{*}\right)^{k}\right)
$$

for all $n, k \in \mathbb{N}$, then $\mu$ is called $*$-distribution of a and is uniquely defined.

The normality of $a$ in part b) of the above definition assures existence of the given integral. If $a$ is selfadjoint, that is a special case of normality, the support of the measure $\mu$ is on $\mathbb{R}$ and hence the integral in Definition 2.1.4 is simplified to (2.1) given below. Conversely, assume that the support of the $*$-distribution of $a$ is real and compact. Then the real probability measure $\mu$, mentioned in Definition 2.1.4, is related to the moments by

$$
\tau\left(a^{k}\right)=\int_{\mathbb{R}} x^{k} d \mu(x)
$$

and is called a distribution of $a$. The distribution of $a \in \mathcal{A}$ defined on a compact support is characterized by its moments $\tau(a), \tau\left(a^{2}\right), \ldots$, see Couillet and Debbah (2011) p. 99.

Definition 2.1.5. The variables $\left(a_{1}, a_{2}, \ldots, a_{m}\right)$ and $\left(b_{1}, \ldots, b_{n}\right)$ are said to be free if and only if for any $\left(P_{i}, Q_{i}\right)_{1 \leq i \leq p} \in\left(\mathbb{C}\left\langle a_{1}, \ldots, a_{m}\right\rangle \times \mathbb{C}\left\langle b_{1}, \ldots, b_{n}\right\rangle\right)^{p}$ such that if

$$
\tau\left(P_{i}\left(a_{1}, \ldots, a_{m}\right)\right)=0, \quad \tau\left(Q_{i}\left(b_{1}, \ldots, b_{n}\right)\right)=0 \quad \forall i=1, \ldots, p
$$

the following equation holds:

$$
\tau\left(\prod_{1 \leq i \leq p} P_{i}\left(a_{1}, \ldots, a_{m}\right) Q_{i}\left(b_{1}, \ldots, b_{n}\right)\right)=0
$$

where $\mathbb{C}\left\langle a_{1}, \ldots, a_{m}\right\rangle$ denotes all polynomials in $m$ non-commutative indeterminants, i.e., symbols that are treated as variables.

To show that freeness does not go with classical independence in Lemma 2.1.2, given below, we first state and prove Lemma 2.1.1, where both lemmas are well known.

Lemma 2.1.1. Let $a$ and $b$ be free elements of a non-commutative probability space $(\mathcal{A}, \tau)$. Then, we have:

$$
\begin{aligned}
\tau(a b) & =\tau(a) \tau(b), \\
\tau(a b a) & =\tau\left(a^{2}\right) \tau(b), \\
\tau(a b a b) & =\tau\left(a^{2}\right) \tau(b)^{2}+\tau(a)^{2} \tau\left(b^{2}\right)-\tau(a)^{2} \tau(b)^{2} .
\end{aligned}
$$


Proof. For free $a$ and $b$ we have

$$
\begin{aligned}
\tau\left(\left(a-\tau(a) 1_{\mathcal{A}}\right)\left(b-\tau(b) 1_{\mathcal{A}}\right)\right) & =0, \\
\tau(a b-a \tau(b)-\tau(a) b+\tau(a) \tau(b)) & =0, \\
\tau(a b) & =\tau(a) \tau(b) .
\end{aligned}
$$

The operations on the elements of algebra are well defined as the algebra is supposed to be closed under addition and multiplication. Then also

$$
\begin{aligned}
\tau\left(\left(a-\tau(a) 1_{\mathcal{A}}\right)\left(b-\tau(b) 1_{\mathcal{A}}\right)\left(a-\tau(a) 1_{\mathcal{A}}\right)\right) & =0, \\
\tau\left((a b-a \tau(b)-\tau(a) b+\tau(a) \tau(b))\left(a-\tau(a) 1_{\mathcal{A}}\right)\right) & =0 \\
\tau(a b a-a \tau(b) a-\tau(a) b a+\tau(a) \tau(b) a-a b \tau(a) & \\
+a \tau(b) \tau(a)+\tau(a) b \tau(a)-\tau(a) \tau(b) \tau(a)) & =0 \\
\tau(a b a)-\tau\left(a^{2}\right) \tau(b)-\tau(a) \tau(b a)+\tau(a) \tau(b) \tau(a)-\tau(a b) \tau(a) & \\
+\tau(a) \tau(b) \tau(a)+\tau(a) \tau(b) \tau(a)-\tau(a) \tau(b) \tau(a) & =0 \\
\tau(a b a) & =\tau\left(a^{2}\right) \tau(b) .
\end{aligned}
$$

Similar calculations show that

$$
\tau(a b a b)=\tau\left(a^{2}\right) \tau(b)^{2}+\tau(a)^{2} \tau\left(b^{2}\right)-\tau(a)^{2} \tau(b)^{2} .
$$

One can prove that freeness and commutativity cannot take place simultaneously as it is now stated in the next lemma.

Lemma 2.1.2. Let $a$ and $b$ be non-trivial elements of the $*$-algebra $\mathcal{A}$, equipped with the functional $\tau$ such that $a$ and $b$ commute, i.e., $a b=b a$. Then, $a$ and $b$ are not free.

Proof. Proof is done by contradiction. Take two non-trivial elements $a$ and $b$ of the $*$-algebra $\mathcal{A}$ such that they are both free and commute. Then

$$
\tau(a b a b) \stackrel{a b=b a}{=} \tau\left(a^{2} b^{2}\right) \stackrel{(2.2)}{=} \tau\left(a^{2}\right) \tau\left(b^{2}\right)
$$

and

$$
\tau(a b a b) \stackrel{(2.3)}{=} \tau\left(a^{2}\right) \tau(b)^{2}+\tau(a)^{2} \tau\left(b^{2}\right)-\tau(a)^{2} \tau(b)^{2} .
$$


These two equalities give

$$
\tau\left(a^{2}\right) \tau(b)^{2}+\tau(a)^{2} \tau\left(b^{2}\right)-\tau(a)^{2} \tau(b)^{2}-\tau\left(a^{2}\right) \tau\left(b^{2}\right)=0
$$

Then, as $a$ and $b$ are free

$$
\begin{aligned}
\tau\left(\left(a-\tau(a) 1_{\mathcal{A}}\right)^{2}\right) \tau\left(\left(b-\tau(b) 1_{\mathcal{A}}\right)^{2}\right) & =\tau\left(a^{2}-2 a \tau(a)+\tau(a)^{2} 1_{\mathcal{A}}\right) \tau\left(b^{2}-2 b \tau(b)+\tau(b)^{2} 1_{\mathcal{A}}\right) \\
& =\left(\tau\left(a^{2}\right)-2 \tau(a)^{2}+\tau(a)^{2}\right)\left(\tau\left(b^{2}\right)-2 \tau(b)^{2}+\tau(b)^{2}\right) \\
& =\left(\tau\left(a^{2}\right)-\tau(a)^{2}\right)\left(\tau\left(b^{2}\right)-\tau(b)^{2}\right) \\
& =\tau\left(a^{2}\right) \tau\left(b^{2}\right)-\tau\left(a^{2}\right) \tau(b)^{2}-\tau(a)^{2} \tau\left(b^{2}\right)+\tau(a)^{2} \tau(b)^{2} \\
& \stackrel{(2.4)}{=} 0 .
\end{aligned}
$$

Then, either $\tau\left(\left(a-\tau(a) 1_{\mathcal{A}}\right)^{2}\right)=0$ or $\tau\left(\left(b-\tau(b) 1_{\mathcal{A}}\right)^{2}\right)=0$. As long as the functional $\tau$ is faithful, i.e., $\tau\left(a^{*} a\right)=0 \Rightarrow a=0$, the obtained equality implies that $a=\tau(a) 1_{\mathcal{A}}$ or $b=\tau(b) 1_{\mathcal{A}}$. We can conclude that at least one of the elements $a$ or $b$ is trivial, which contradicts the assumption that the equations hold for any non-trivial elements, which proves the statement.

\subsubsection{Space $\left(R M_{p}(\mathbb{C}), \tau\right)$}

In this subsection we consider a particular example of a non-commutative space $\left(R M_{p}(\mathbb{C}), \tau\right)$. Let $(\Omega, \mathcal{F}, P)$ be a probability space, then $R M_{p}(\mathbb{C})$ denotes the set of all $p \times p$ random matrices, with entries which belong to $\bigcap_{p=1,2, \ldots} L^{p}(\Omega, P)$, i.e., entries which are complex random variables with finite moments of any order. Defined in this way $R M_{p}(\mathbb{C})$ is a $*$-algebra, with the classical matrix product as multiplication and the conjugate transpose as $*-$ operation. The $*-$ algebra is equipped with tracial functional $\tau$ defined as the expectation of the normalized trace $\operatorname{Tr}_{p}$ in the following way

$$
\tau(X):=\mathbb{E}\left(\operatorname{Tr}_{p}(X)\right)=\mathbb{E}\left(\frac{1}{p} \operatorname{Tr}(X)\right)=\frac{1}{p} \mathbb{E}\left(\sum_{i=1}^{p} X_{i i}\right)=\frac{1}{p} \sum_{i=1}^{p} \mathbb{E}\left(X_{i i}\right),
$$

where $X=\left(X_{i j}\right)_{i, j=1}^{p} \in R M_{p}(\mathbb{C})$.

The form of the chosen functional $\tau$ is determined by the fact that the distribution of the eigenvalues is of particular interest to us. Notice that for any normal matrix $X \in\left(R M_{p}(\mathbb{C}), \tau\right)$, i.e., for any Hermitian matrix (i.e., $X=X^{*}$ ), the eigenvalue distribution $\mu_{X}$ is the $*$-distribution with respect to a given functional $\tau$ defined in Definition 2.1.4. Furthermore, $\mu_{X}$ is supported on the real line.

Now, consider a matrix $X$ of size $p \times p$ with eigenvalues $\lambda_{1}, \ldots, \lambda_{p}$. Then

$$
\operatorname{Tr}_{p}\left(X^{k}\left(X^{*}\right)^{n}\right)=\frac{1}{p} \sum_{i=1}^{p} \lambda_{i}^{k}{\overline{\lambda_{i}}}^{n}=\int_{\mathbb{C}} z^{k} \bar{z}^{n} d \mu_{X}(z)
$$


for all $k, n \in \mathbb{N}$, where $\mu_{X}$ is a spectral probability measure, corresponding to the normalized spectral distribution function defined as

$$
F_{p}^{X}(x)=\frac{1}{p} \sum_{k=1}^{p} \mathbb{1}_{\left\{\lambda_{k} \leq x\right\}}, \quad x \geq 0,
$$

where $\mathbb{1}_{\left\{\lambda_{k} \leq x\right\}}$ stands for the indicator function, i.e.,

$$
\mathbb{1}_{\left\{\lambda_{k} \leq x\right\}}= \begin{cases}1 & \text { for } x \leq \lambda_{k} \\ 0 & \text { otherwise }\end{cases}
$$

For

$$
\mu_{X}(x)=\frac{1}{p} \int_{\Omega} \sum_{k=1}^{p} \delta_{\lambda_{k}(\omega)} d P(\omega),
$$

where $\delta_{\lambda_{k}(\omega)}$ stands for Dirac delta, we obtain a generalization of the above statement for $X$ being a normal random matrix, so

$$
\tau\left(X^{k}\left(X^{*}\right)^{n}\right)=\frac{1}{p} \int_{\Omega} \sum_{i=1}^{p} \lambda_{i}^{k}(\omega){\overline{\lambda_{i}(\omega)}}^{n} d P(\omega)=\int_{\mathbb{C}} z^{k} \bar{z}^{n} d \mu_{X}(z) .
$$

Hence, trace $\tau$ as defined in (2.5) is related to the spectral probability measure $\mu_{X}$, which is $*$-distribution in the sense given by Definition 2.1.4b.

In this thesis we mostly consider positive definite (symmetric) matrices. Then, if $X$ is Hermitian then all the eigenvalues are real and $\mu_{X}$ is related to $\tau\left(X^{k+n}\right)$ by

$$
\tau\left(X^{k+n}\right)=\int_{\mathbb{R}} x^{k+n} d \mu_{X}(x)
$$

\subsubsection{Asymptotic freeness}

The concept of asymptotic freeness was established by Voiculescu (1991), whose work discussed and proved the asymptotical freeness of Gaussian random matrices and constant diagonal matrices. The main result was given for the Gaussian Unitary Ensemble (GUE), where an ensemble of random matrices is a family of random matrices with a density function that expresses the probability density $f$ of any member of the family to be observed. There $H_{n} \rightarrow U H_{n} U^{-1}$ is a transformation which leaves $f\left(H_{n}\right)$ invariant, $U$ is a unitary matrix (i.e., $U U^{*}=U^{*} U=I$ ) and the matrices $H_{n}$ are Hermitian.

Theorem 2.1.1 (Voiculescu's Asymptotic Freeness). Let $X_{p, 1}, X_{p, 2}, \ldots$ be independent (in the classical sense) $p \times p$ GUE. Then there exists a functional $\phi$ in some non-commutative polynomials of $X_{1}, X_{2}, \ldots$ with complex coefficients such that 
- $\left(X_{p, 1}, X_{p, 2}, \ldots\right)$ has a limiting distribution $\phi$ as $p \rightarrow \infty$, i.e.,

$$
\phi\left(X_{i_{1}} X_{i_{2}} \cdots X_{i_{k}}\right)=\lim _{p \rightarrow \infty} \tau\left(X_{p, i_{1}} X_{p, i_{2}} \cdots X_{p, i_{k}}\right),
$$

for all $i_{j} \in \mathbb{N}, j \in \mathbb{N}$, where $\tau(X)=\mathbb{E}\left(\operatorname{Tr}_{p} X\right)$.

- $X_{1}, X_{2}, \ldots$ are freely independent with respect to $\phi$, see Definition 2.1.5.

Voiculascu's work was followed by Dykema (1993) who replaced the Gaussian entries of the matrices with more general non-Gaussian random variables. Furthermore, the constant diagonal matrices were generalized to some constant block diagonal matrices, such that the block size remains constant. In general random matrices with independent entries of size $p \times p$ tend to be asymptotically free when $p \rightarrow \infty$, under certain conditions.

To give some additional examples of asymptotically free pairs of matrices, note that two independent Haar distributed unitary $p \times p$ matrices are asymptotically free as $p \rightarrow \infty$. Moreover, two i.i.d. $p \times p$ Gaussian distributed random matrices are asymptotically free as $p \rightarrow \infty$. We also observe that asymptotic freeness holds between i.i.d. Wigner matrices, i.e., matrices of the form $\left(X+X^{*}\right) / 2$, where $X$ is a $p \times p$ matrix with i.i.d. Gaussian entries. This was proven by Dykema (1993). Asymptotic free independence also holds for Gaussian and Wishart random matrices and for Wigner and Wishart matrices, according to Capitaine and Donati-Martin (2007).

Following Müller (2002) we want to point out that there exist matrices which are dependent (in a classical sense) and asymptotically free, as well as matrices with independent entries (in a classical sense), which are not asymptotically free.

\subsection{Combinatorial interpretation of freeness and free cumulants}

Combinatorial interpretation of freeness, described using free cumulants (see, Definition 2.2.3 given below) was established by Speicher (1994) and was further developed by Nica and Speicher (2006). Free cumulants play an important role in Chapter 3, where the $\mathrm{R}$-transform is defined, and in the papers of the second part of the thesis.

Definition 2.2.1. Let $\mathcal{V}=V_{1}, \ldots, V_{p}$ be a partition of the set $\{1, \ldots, r\}$, i.e., for all $i=1, \ldots, p$ the $V_{i}$ are ordered and disjoint sets and $\bigcup_{i=1}^{p} V_{i}=\{1, \ldots, r\}$. The $\mathcal{V}$ is called non-crossing if for all $i, j=1, \ldots, p$ with $V_{i}=\left(v_{1}, \ldots, v_{n}\right)$ (such that $\left.v_{1}<\ldots<v_{n}\right)$ and $V_{j}=\left(w_{1}, \ldots, w_{m}\right)$ (such that $w_{1}<\ldots<w_{m}$ ) we have

$$
w_{k}<v_{1}<w_{k+1} \Leftrightarrow w_{k}<v_{n}<w_{k+1} \quad \text { for } k=1, \ldots, m-1 \text {. }
$$


Presented here is the definition of the non-crossing partition, according to Speicher, which can, equivalently to Definition 2.2.1, be given in a recursive form as below.

Definition 2.2.2. The partition $\mathcal{V}=\left\{V_{1}, \ldots, V_{p}\right\}$ is non-crossing if at least one of the $V_{i}$ is a segment of $(1, \ldots, r)$, i.e., it has the form $V_{i}=(k, k+1, \ldots, k+$ $m$ ) and the remaining partition $\left\{V_{1}, \ldots, V_{i-1}, V_{i+1}, \ldots, V_{p}\right\}$ is a non-crossing partition of $\{1, \ldots, r\} \backslash V_{i}$.

Let the set of all non-crossing partitions over $\{1, \ldots, r\}$ be denoted by $N C(r)$.

Note that in the following recursive definition of the cumulants the square brackets are used to denote the cumulants with respect to the partitions, while the parentheses are used for the cumulants of some set of variables.

Definition 2.2.3. Let $(\mathcal{A}, \tau)$ be a non-commutative probability space. Then we define the cumulant functionals $k_{k}: \mathcal{A}^{k} \rightarrow \mathbb{C}$, for all $i \in \mathbb{N}$ by the momentcumulant relation

$$
k_{1}(a)=\tau(a), \quad \tau\left(a_{1} \cdots a_{k}\right)=\sum_{\pi \in N C(k)} k_{\pi}\left[a_{1}, \ldots, a_{k}\right]
$$

where the sum is taken over all non-crossing partitions of the set $\left\{a_{1}, a_{2}, \ldots, a_{k}\right\}$. Moreover, $a_{1} \cdots a_{k}$ denotes the product of all elements $a_{i}$ for $i=1, \ldots, k$ and

$$
\begin{aligned}
k_{\pi}\left[a_{1}, \ldots, a_{k}\right] & =\prod_{i=1}^{r} k_{V(i)}\left[a_{1}, \ldots, a_{k}\right] & \pi & =\{V(1), \ldots, V(r)\}, \\
k_{V}\left[a_{1}, \ldots, a_{k}\right] & =k_{s}\left(a_{v(1)}, \ldots, a_{v(s)}\right) & V & =(v(1), \ldots, v(s)) .
\end{aligned}
$$

For the element a of a non-commutative algebra $(\mathcal{A}, \tau)$ we define the cumulant of a as

$$
k_{n}^{a}=k_{n}(a, \ldots, a)
$$

We illustrate the definition by considering cumulants on a two-element set $\left\{a_{1}, a_{2}\right\}$, such that $a_{1}, a_{2}$ belong to a non-commutative probability space equipped with the tracial functional $\tau$. Then, $k_{1}\left(a_{i}\right)=\tau\left(a_{i}\right)$ for $i=1,2$. The only non-crossing partitions of the two-element set are the segments $\left\{a_{1}, a_{2}\right\}$ and $\left\{a_{1}\right\},\left\{a_{2}\right\}$ so $\tau\left(a_{1} a_{2}\right)=\sum_{\pi \in N C(2)} k_{\pi}\left[a_{1}, a_{2}\right]=k_{2}\left(a_{1}, a_{2}\right)+k_{1}\left(a_{1}\right) k_{1}\left(a_{2}\right)=$ $k_{2}\left(a_{1}, a_{2}\right)+\tau\left(a_{1}\right) \tau\left(a_{2}\right)$. Hence, $k_{2}\left(a_{1}, a_{2}\right)=\tau\left(a_{1} a_{2}\right)-\tau\left(a_{1}\right) \tau\left(a_{2}\right)$ is a cumulant of the two-element set $\left\{a_{1}, a_{2}\right\}$, while $k_{\pi}\left[a_{1}, a_{2}\right]$ denotes the cumulant of the partition $\pi$.

Lemma 2.2.1. The cumulants given by Definition 2.2.3 are well defined (statement is unambiguous).

Proof. Following the definition of a cumulant

$$
\tau\left(a_{1}, \ldots, a_{n}\right)=\sum_{\pi \in N C(n)} k_{\pi}\left[a_{1}, \ldots, a_{n}\right]=k_{n}\left(a_{1}, \ldots, a_{n}\right)+\sum_{\pi \in N C(n), \pi \neq 1_{n}} k_{\pi}\left[a_{1}, \ldots, a_{n}\right],
$$


where $\pi \neq 1_{n}$ means that we consider partitions which are different from the $n$-elements segment, i.e., $\pi \neq\{1,2, \ldots, n\}$. Now the statement of the lemma follows by induction.

To show linearity of cumulants for the sum of free random variables, we need to state a theorem about vanishing mixed cumulants.

Theorem 2.2.1. Let $a_{1}, a_{2}, \ldots, a_{n} \in \mathcal{A}$ then elements $a_{1}, a_{2}, \ldots, a_{n}$ are freely independent if and only if all mixed cumulants vanish, i.e., for $n \geq 2$ and any choice of $i_{1}, \ldots, i_{k} \in\{1, \ldots, n\}$ if there exist $j, k$ such that $j \neq k$, but $i_{j}=i_{k}$ then

$$
k_{n}\left(a_{i_{1}}, \ldots, a_{i_{n}}\right)=0
$$

Proof. The proof can be found in Nica and Speicher (2006).

Now an important theorem is stated which was used in Paper I while considering the $\mathrm{R}$-transform of a sum of matrices.

Theorem 2.2.2. If $a, b \in \mathcal{A}$ be free, then

$$
k_{n}^{a+b}=k_{n}^{a}+k_{n}^{b}, \quad \text { for } n \geq 1 .
$$

Proof. The proof of the theorem follows from the fact that mixed cumulants for free random variables equal zero, see Theorem 2.2.1. Then

$$
k_{n}^{a+b}:=k_{n}(a+b, a+b, \ldots, a+b)=k_{n}(a, a, \ldots, a)+k_{n}(b, b, \ldots, b) .
$$

\subsection{Free moment-cumulant formulas}

There are a number of results regarding a moment-cumulant formula. The most well-known is the relation given in Definition 2.2.3, i.e.,

$$
k_{1}(a)=\tau(a), \quad \tau\left(a_{1} \cdots a_{k}\right)=\sum_{\pi \in N C(k)} k_{\pi}\left[a_{1}, \ldots, a_{k}\right] .
$$

This recursive formula uses summation of free cumulants over the non-crossing partitions of the set $\{1, \ldots, k\}$. Another way to look at free cumulants is by using the Möbius function as well as non-crossing partitions:

$$
k_{\pi}\left[a_{1}, \ldots, a_{k}\right]=\sum_{\sigma \in N C(k), \sigma \leq \pi} \tau_{\sigma}\left[a_{1}, \ldots, a_{k}\right] \mu(\sigma, \pi),
$$


where $\tau_{k}\left(a_{1}, \ldots, a_{k}\right):=\tau\left(a_{1}, \ldots, a_{k}\right), \tau_{\pi}\left[a_{1}, \ldots, a_{k}\right]:=\prod_{V \in \pi} \tau_{V}\left[a_{1}, \ldots, a_{k}\right]$ and $\mu$ is the Möbius function on $N C(k)$. Equivalently, this formula can also be given in the form

$$
k_{n}\left(a_{1}, \ldots, a_{n}\right)=\sum_{\sigma \in N C(n)} \tau_{\sigma}\left[a_{1} \cdots a_{n}\right] \mu\left(\sigma, 1_{n}\right),
$$

where $1_{n}=\{1, \ldots, n\}$, so it is a maximal element of a poset (partially ordered set) $N C(n)$, or a so-called $n$-elements segment. For more details about the above formulations see Nica and Speicher (2006) and Speicher (1994).

Let $\left\{k_{i}\right\}_{i=1}^{\infty}$ be the free cumulants and $\left\{m_{i}\right\}_{i=1}^{\infty}$ be the free moments for an element of a non-commutative probability space. Then the following non-recursive relation between free moments and free cumulants has been shown by Mottelson (2012) together with a proof which is based on the Lagrange inversion formula and is inspired by the work of Haagerup (1997).

$$
\begin{aligned}
k_{p} & =m_{p}+\sum_{j=2}^{p} \frac{(-1)^{j-1}}{j}\left(\begin{array}{c}
p+j-2 \\
j-1
\end{array}\right) \sum_{Q_{j}} m_{q_{1}} \cdots m_{q_{j}}, \\
m_{p} & =k_{p}+\sum_{j=2}^{p} \frac{1}{j}\left(\begin{array}{c}
p \\
j-1
\end{array}\right) \sum_{Q_{j}} k_{q_{1}} \cdots k_{q_{j}},
\end{aligned}
$$

where $Q_{j}=\left\{\left(q_{1}, q_{2}, \ldots, q_{j}\right) \in \mathbb{N}^{j} \mid \sum_{i=1}^{j} q_{i}=p\right\}$ and $k_{p}\left(m_{p}\right)$ denotes the $p$ th free cumulant ( $p$ th free moment, respectively) of some element of a non-commutative space.

In Paper II free cumulants and moments are related as follows:

$$
k_{t}=\sum_{i=1}^{t}(-1)^{i+1}\left(\begin{array}{c}
\mathbf{m}, i,> \\
t
\end{array}\right)-\sum_{h=2}^{t-1} k_{h}\left(\begin{array}{c}
\mathbf{m}, h-1, \geq \\
t-h
\end{array}\right) .
$$

The result was obtained using a derivation based on the concepts of Stieltjes and $\mathrm{R}$-transforms and differs from alternatives by not using non-crossing partitions.

Note that we introduce a shortened notation for the sum of products of $h$ moments, where each of the moments has a degree given by the index $i_{k}$, $k=1, \ldots, h$, the sum of indexes $i_{1}+i_{2}+\ldots+i_{h}=t$ and each index $i_{k} \succ 0$, where $\succ$ stands for the partial order relation

$$
\left(\begin{array}{c}
\mathbf{m}, h, \succ \\
t
\end{array}\right)=\sum_{\substack{i_{1}+i_{2}+\ldots+i_{h}=t \\
\forall k}} m_{i_{1}} m_{i_{2}} \cdot \ldots \cdot m_{i_{h}} .
$$

Let us present an example comparing computations for the 5th free cumulant based on the summation over non-crossing partitions (Definition 2.2.3), as well as over the non-negative partitions of the number presented in (2.8). 
Example 2.3.1. The calculations of the fifth free cumulant, by use of Definition 2.2.3, require a summation over $N C(5)$. Consider the crossing partitions of the set $\{1,2,3,4,5\}$. Then

$$
\begin{aligned}
m_{5}= & \sum_{\pi \in N C(5)} k_{\pi}[a, a, a, a, a]=k_{5}+5 k_{4} k_{1}+\left(\left(\begin{array}{l}
5 \\
2
\end{array}\right)-5\right) k_{3} k_{2} \\
& +\left(\begin{array}{l}
5 \\
3
\end{array}\right) k_{3} k_{1}^{2}+\left(\left(\begin{array}{l}
5 \\
1
\end{array}\right) \frac{1}{2}\left(\begin{array}{l}
4 \\
2
\end{array}\right)-5\right) k_{2}^{2} k_{1}+\left(\begin{array}{l}
5 \\
2
\end{array}\right) k_{2} k_{1}^{3}+k_{1}^{5} \\
= & k_{5}+5 k_{4} k_{1}+5 k_{3} k_{2}+10 k_{3} k_{1}^{2}+10 k_{2}^{2} k_{1}+10 k_{2} k_{1}^{3}+k_{1}^{5}, \\
k_{5}= & m_{5}-5 k_{4} k_{1}-5 k_{3} k_{2}-10 k_{3} k_{1}^{2}-10 k_{2}^{2} k_{1}-10 k_{2} k_{1}^{3}-k_{1}^{5} \\
= & m_{5}-5\left(m_{4}-4 m_{3} m_{1}-2 m_{2}^{2}+10 m_{2} m_{1}^{2}-5 m_{1}^{4}\right) m_{1} \\
& -5\left(m_{3}-3 m_{2} m_{1}+2 m_{1}^{3}\right)\left(m_{2}-m_{1}^{2}\right)-10\left(m_{3}-3 m_{2} m_{1}+2 m_{1}^{3}\right) m_{1}^{2} \\
& -10\left(m_{2}-m_{1}^{2}\right)^{2} m_{1}-10\left(m_{2}-m_{1}^{2}\right) m_{1}^{3}-m_{1}^{5} \\
= & m_{5}-5 m_{4} m_{1}+15 m_{3} m_{1}^{2}+15 m_{2}^{2} m_{1}-35 m_{2} m_{1}^{3}-5 m_{3} m_{2}+14 m_{1}^{5}
\end{aligned}
$$

as the crossing partitions given in Figure 2.1 should not be included in the above sums.

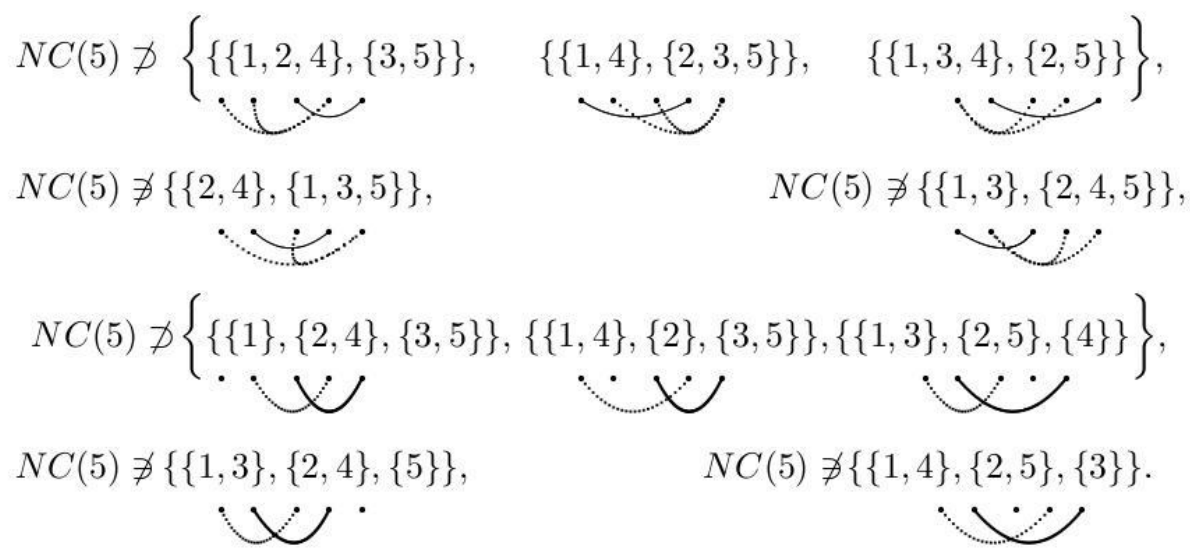

Figure 2.1: Crossing partitions of the set $\{1,2,3,4,5\}$.

When using (2.8)

$$
\begin{aligned}
k_{5} & =\sum_{i=1}^{5}(-1)^{i+1} \sum_{\substack{j_{1}+\ldots+j_{i}=5 \\
\forall k j_{k}>0}} m_{j_{1}} \cdot \ldots \cdot m_{j_{i}}-\sum_{h=2}^{4} k_{h} \sum_{\substack{j_{1}+\ldots+j_{h-1}=5-h \\
\forall k j_{j} \geq 0}} m_{j_{1}} \cdot \ldots \cdot m_{j_{h-1}} \\
& =m_{5}-5 m_{4} m_{1}-5 m_{3} m_{2}+15 m_{3} m_{1}^{2}+15 m_{2}^{2} m_{1}-35 m_{2} m_{1}^{3}+14 m_{1}^{5} .
\end{aligned}
$$

The calculations using both methods are presented. To some extent we find that summation over the $i_{1}, \ldots, i_{h}$, such that $i_{1}+\ldots+i_{h}=k$ is simpler than sum- 
mation over non-crossing partitions. Moreover, there is a strong belief that the results of Paper II can, within free probability, successfully complete already existing knowledge regarding cumulant-moment relations, and in some particular cases replace previously used formulas in order to provide easier calculations. 


\section{Stieltjes and $\mathrm{R}$-transform}

The Stieltjes transform is commonly used in research regarding the spectral measure of random matrices. It appears, among others, in formulations and proofs of a number of results published within Random matrix theory, i.e., Marčenko and Pastur (1967), Girko and von Rosen (1994), Silverstein and Bai (1995), Hachem et al. (2007). Thanks to good algebraic properties, such as being an analytic function on the complex complement of support of the measure or bounded in any point $z$ by $1 / \Im z$ (where $\Im z$ stands for the imaginary part of the complex number $z$ ), it often simplifies calculations for obtaining the limit of empirical spectral distribution for large dimensional random matrices. More specifically, the Stieltjes transform provides the exact method to describe the support of the corresponding measure. The last mentioned property is often used in signal processing for signal detection.

The second section of this chapter presents the $\mathrm{R}$-transform introduced within free probability theory and strongly related to the Stieltjes transform. The $\mathrm{R}$-transform provides a way to obtain an analytical form of the asymptotic distribution of eigenvalues for the sums of certain random matrices. More specifically, we will consider the distribution function of the matrix quadratic form $Q_{n}=\frac{1}{n} X_{1} X_{1}^{\prime}+\ldots+\frac{1}{n} X_{k} X_{k}^{\prime}$, where $X_{i}$ follows the matrix normal distribution. An asymptotic distribution of $Q_{n}$ for $k=1$, under some additional assumptions on $X_{1}$, is given by the Marčenko-Pastur law. For $k=2$ it is described by a system of equations given in Girko and von Rosen (1994) and arbitrary $k$ is analyzed in Paper I.

Both the Stieltjes and R-transforms and their properties are discussed to different extents by Nica and Speicher (2006) (where, after a short introduction to the Stieltjes transform, its relation to the R-transform is investigated), Couillet and Debbah (2011) (where application to random matrix theory is discussed and the properties of the Stieltjes transform are given), by Hiai and Petz (2000) (extensive study of Stieltjes and R-transforms) and within the lecture notes of Manjunath (2011). A version of the Stieltjes transform, the Cauchy transform, is also described in Cima et al. (2006). 


\subsection{Stieltjes transform}

The Stieltjes transform defined in this section, up to a sign, is often referred to as the Cauchy transform (i.e., Cima et al., 2006; Hiai and Petz, 2000; Nica and Speicher, 2006) or the Stieltjes-Cauchy transform (i.e., Hasebe, 2012; Bożejko and Demni, 2009). In this thesis we use the term Stieltjes transform following the work by Couillet and Debbah (2011).

Definition 3.1.1. Let $\mu$ be a non-negative, finite borel measure on $\mathbb{R}$, e.g. a probability measure. Then we define the Stieltjes transform of $\mu$ by

$$
G(z)=\int_{\mathbb{R}} \frac{1}{z-x} d \mu(x),
$$

for all $z \in\{z: z \in \mathbb{C}, \Im(z)>0\}$, where $\Im(z)$ denotes the imaginary part of $z$.

Remark 3.1.1. Note that for $z \in\{z: z \in \mathbb{C}, \Im(z)>0\}$ the Stieltjes transform is well defined and $G(z)$ is analytical for all $z \in\{z: z \in \mathbb{C}, \Im(z)>0\}$.

Proof. The fact that the Stieltjes transform is well defined follows from the fact that for the domain the function $\frac{1}{z-x}$ is bounded.

One can show that $G(z)$ is analytical for all $z \in\{z: z \in \mathbb{C}, \Im(z)>0\}$ using Morera's theorem (see work by Greene and Krantz, 2006). Then, it is enough to show that the contour integral $\oint_{\Gamma} G(z) d z=0$, for all closed contours $\Gamma$ in $z \in\{z: z \in \mathbb{C}, \Im(z)>0\}$. We are allowed to interchange integrals and obtain

$$
\int_{\mathbb{R}} \oint_{\Gamma} \frac{1}{z-x} d z d \mu(x)=\int_{\mathbb{R}} 0 d \mu(x)=0,
$$

where as $\frac{1}{z-x}$ is analytic the inner integral vanishes by Cauchy's integral theorem for any closed contour $\Gamma$.

The above definition can be extended to all $z$ that does not belong to the support of the measure $\mu$. Although extension is possible, we will consider the domain of the transform to be restricted to the upper half plane of $\mathbb{C}$ since we want $G(z)$ to be analytical.

Now, we introduce the Stieltjes inversion formula, which allows us to use knowledge about the transform $G$ to derive the measure $\mu$.

Theorem 3.1.1. For any open interval $I=(a, b)$, such that neither $a$ nor $b$ are atoms for the probability measure $\mu$, the Stieltjes inversion formula is given by

$$
\mu(I)=-\frac{1}{\pi} \lim _{y \rightarrow 0} \int_{I} \Im G(x+\mathrm{i} y) d x .
$$


Proof. The proof is a detailed version of the proof given in e.g. Couillet and Debbah (2011). We have

$$
\begin{aligned}
-\frac{1}{\pi} \lim _{y \rightarrow 0} \int_{I} \Im G(x+\mathrm{i} y) & d x=-\frac{1}{\pi} \lim _{y \rightarrow 0} \int_{I} \int_{\mathbb{R}} \Im \frac{1}{x+\mathrm{i} y-t} d \mu(t) d x \\
& \stackrel{(*)}{=} \frac{1}{\pi} \lim _{y \rightarrow 0} \int_{\mathbb{R}} \int_{a}^{b} \frac{y}{(t-x)^{2}+y^{2}} d x d \mu(t) \\
= & \frac{1}{\pi} \lim _{y \rightarrow 0} \int_{\mathbb{R}} \arctan \left(\frac{b-t}{y}\right)-\arctan \left(\frac{a-t}{y}\right) d \mu(t) \\
& \stackrel{(\diamond)}{=} \frac{1}{\pi} \int_{\mathbb{R}} \lim _{y \rightarrow 0}\left(\arctan \left(\frac{b-t}{y}\right)-\arctan \left(\frac{a-t}{y}\right)\right) d \mu(t) .
\end{aligned}
$$

The order of integration can be interchanged in $(*)$ due to continuity of the function $\frac{y}{(t-x)^{2}+y^{2}}$. Interchanging of order between integration and taking the limit in $(\diamond)$ follows by the Bounded convergence theorem as $\mu(\mathbb{R}) \leq 1<\infty$ and

$$
\exists M \text { such that } \arctan \left(\frac{b-t}{y}\right)-\arctan \left(\frac{a-t}{y}\right)<M, \quad \forall y \quad \forall t
$$

so it is a uniformly bounded real-valued measurable function for all $y$.

Then, using that $\lim _{y \rightarrow 0} \arctan \left(\frac{T}{y}\right)=\frac{\pi}{2} \operatorname{sgn}(T)$ for $T \in \mathbb{R}$ we get

$$
\arctan \left(\frac{b-t}{y}\right)-\arctan \left(\frac{a-t}{y}\right) \stackrel{y \rightarrow 0}{\longrightarrow} \begin{cases}0 & \text { if } t<a \text { or } t>b \\ \frac{\pi}{2} 2=\pi & \text { if } t \in(a, b)\end{cases}
$$

which, by the Dominated convergence theorem, completes the proof.

More generally, the statement of Theorem 3.1.1 for any $\mu$ being a probability measure on $\mathbb{R}$ and any $a<b$ becomes

$$
\mu((a, b))+\frac{1}{2} \mu(\{a\})+\frac{1}{2} \mu(\{b\})=-\frac{1}{\pi} \lim _{y \rightarrow 0} \int_{I} \Im G(x+\mathrm{i} y) d x .
$$

For further reading, see Manjunath (2011).

Theorem 3.1.2. Let $\mu_{n}$ be a sequence of probability measures on $\mathbb{R}$ and let $G_{\mu_{n}}$ denote the Stieltjes transform of $\mu_{n}$. Then:

a) if $\mu_{n} \rightarrow \mu$ weakly, where $\mu$ is a measure on $\mathbb{R}$, then $G_{\mu_{n}}(z) \rightarrow G_{\mu}(z)$ pointwise for any $z \in\{z: z \in \mathbb{C}, \Im(z)>0\}$.

b) if $G_{\mu_{n}}(z) \rightarrow G(z)$ pointwise, for all $z \in\{z: z \in \mathbb{C}, \Im(z)>0\}$ then there exists a unique non-negative and finite measure such that $G=G_{\mu}$ and $\mu_{n} \rightarrow \mu$ weakly. 
Proof.

a) We know that if $\mu_{n} \rightarrow \mu$, then for all bounded and continuous functions $f$ the following

$$
\int f d \mu_{n} \rightarrow \int f d \mu
$$

holds. As $f(x)=\frac{1}{z-x}$ is both bounded and continuous outside of $\mathbb{R}$ for all fixed $z \in\{z: z \in \mathbb{C}, \Im(z)>0\}$ we conclude that

$$
G_{\mu_{n}}(z)=\int_{\mathbb{R}} \frac{1}{z-x} d \mu_{n}(x) \rightarrow \int_{\mathbb{R}} \frac{1}{z-x} d \mu(x)=G_{\mu}(z)
$$

pointwise.

b) Now, assume that $G_{\mu_{n}}(z) \rightarrow G(z)$ pointwise. As $\mu_{n}$ is a probability measure (so it is bounded and a positive measure for which $\sup _{n} \mu_{n}(\mathbb{R})<\infty$ ), then by Helly's selection principle $\mu_{n}$ has a weakly convergent subsequence. That subsequence is denoted by $\mu_{n_{k}}$ and its limit by $\mu$.

As $f(x)=\frac{1}{z-x}$ is bounded, continuous outside of $\mathbb{R}$ and $f(x) \stackrel{x \rightarrow \pm \infty}{\longrightarrow} 0$, by part a) $G_{\mu_{n_{k}}}(z) \rightarrow G_{\mu}(z)$ pointwise for all $z \in\{z: z \in \mathbb{C}, \Im(z)>0\}$. Then $G_{\mu}=G$, which means that for all converging subsequences we obtain the same limit $\mu$, since the inverse Stieltjes transform is unique. Hence, $\mu_{n} \rightarrow \mu$.

In the last part of this section we state a lemma which relates the Stieltjes transform to the moment generating function. It will be later used when proving a relation between the Stieltjes and $\mathrm{R}$-transform.

Theorem 3.1.3. Let $\mu$ be a probability measure on $\mathbb{R}$ and $\left\{m_{k}\right\}_{k=1, \ldots}$ a sequence of moments (i.e., $m_{k}(\mu)=\int_{\mathbb{R}} t^{k} d \mu(t)$ ). Then, the moment generating function $M^{\mu}(z)=\sum_{k=0}^{\infty} m_{k} z^{k}$ converges to an analytic function in some neighborhood of 0 . For sufficiently large $|z|$

$$
G(z)=\frac{1}{z} M^{\mu}\left(\frac{1}{z}\right) .
$$

holds.

\subsubsection{Stieltjes transform approach for random matrices}

We will often consider a $\mu$ in Definition 3.1.1 being a distribution function. Then, by the uniqueness of the Stiletjes inversion formula we can analyze the distribution function through its Stieltjes transform. 
Consider now an element of the non-commutative space of Hermitian random matrices over the complex plane $X \in R M_{p}(\mathbb{C})$ and note that analyzing the Stieltjes transform is actually simplified to consideration of diagonal elements of the matrix $\left(z I_{p}-X\right)^{-1}$ as

$$
G_{\mu}(z)=\int_{\mathbb{R}} \frac{1}{z-x} d \mu(x)=\frac{1}{p} \operatorname{Tr}\left(z I_{p}-X\right)^{-1},
$$

where $\mu$ denotes the empirical spectral distribution of the matrix $X$. The same holds for the real symmetric random matrices.

Lemma 3.1.1. Let $X$ be a random matrix of size $p \times n$ with complex entries and $z \in\{z: z \in \mathbb{C} \Im(z)>0\}$. Then,

$$
\frac{n}{p} G_{\mu_{X^{*} X}}(z)=G_{\mu_{X X^{*}}}(z)-\frac{p-n}{p z} .
$$

Proof. As $X$ is of size $p \times n$, the matrix $X^{*} X$ is of size $n \times n$ and the matrix $X X^{*}$ is of size $p \times p$. Moreover, $X X^{*}$ and $X^{*} X$ are Hermitian. Assume that $p>n$. Then, $X^{*} X$ has $n$ eigenvalues, while the set of eigenvalues of the matrix $X X^{*}$ consists of the same $n$ eigenvalues and additional $p-n$ zeros. Then, by the definition of the Stieltjes transform, we have $p-n$ times the term $\frac{1}{z-0}=\frac{1}{z}$ and

$$
G_{\mu_{X X^{*}}}(z)=\frac{n}{p} G_{\mu_{X^{*} X}}(z)+\frac{p-n}{p} \frac{1}{z} .
$$

If $p<n$, we have

$$
\begin{aligned}
G_{\mu_{X^{*} X}}(z) & =\frac{p}{n} G_{\mu_{X X^{*}}}(z)+\frac{n-p}{n} \frac{1}{z}, \\
\frac{n}{p} G_{\mu_{X^{*} X}}(z) & =G_{\mu_{X X^{*}}}(z)+\frac{n-p}{p} \frac{1}{z}
\end{aligned}
$$

and the proof is complete.

\section{$3.2 \mathrm{R}$-transform}

The $\mathrm{R}$-transform plays the same role in free probability theory as the logarithm of the Fourier transform (cumulant generating function) in classical probability theory and it is defined in the following way.

Definition 3.2.1 (R-transform). Let $\mu$ be a probability measure with compact support, with $\left\{k_{i}\right\}_{i=1, \ldots}$ being the sequence of cumulants, defined in Chapter 2, Definition 2.2.3. Then the R-transform is given by

$$
R(z)=\sum_{i=0}^{\infty} k_{i+1} z^{i} .
$$


Note that, defined in this way, the $\mathrm{R}$-transform and cumulants $\left\{k_{i}\right\}$ essentially give us the same information. For the compactly supported measure, both cumulants and $\mathrm{R}$-transform carry full information about the underling probability measure.

There is a relation between the $\mathrm{R}$ - and Stieltjes transform $G$, or more precisely $G^{-1}$, which is the inverse with respect to composition, and is often considered as an equivalent definition to Definition 3.2.1. We give a theorem following the book by Tulino and Verdu (2004).

Theorem 3.2.1. Let $\mu$ be a probability measure with compact support, $G(z)$ the Stieltjes transform, and $R(z)$ the $R$-transform. Then,

$$
R(z)=G^{-1}(z)-\frac{1}{z}
$$

The relation between the moment and cumulant generating functions is given by Lemma 3.2.1. This tool is stated here due to its use in the proof of Theorem 3.2.1. Moreover, note that in Lemma 3.2.1 the free cumulant-moment relation formulas are stated, see Section 2.3, and note that the moment generating function will be utilized in Paper II.

Lemma 3.2.1. Let $\left\{m_{i}\right\}_{i \geq 1}$ and $\left\{k_{i}\right\}_{i \geq 1}$ be sequences of complex numbers, with corresponding formal power series

$$
M(z)=1+\sum_{i=1}^{\infty} m_{i} z^{i} \quad C(z)=1+\sum_{i=1}^{\infty} k_{i} z^{i}
$$

as generating functions, such that

$$
m_{i}=\sum_{\pi \in N C(i)} k_{\pi}
$$

where $N C(i)$ stands for the non-crossing partitions over $\{1, \ldots, i\}$. Then,

$$
C(z M(z))=M(z) .
$$

The proof of the lemma has been presented by Nica and Speicher (2006), who used combinatorial tools.

Proof. [Proof of Theorem 3.2.1]

$$
R(G(z))+\frac{1}{G(z)}=z
$$

is equivalent with the statement given in the theorem.

Let $\left\{m_{k}\right\}_{k=1, \ldots}$ and $\left\{k_{i}\right\}_{i=1, \ldots}$ denote the sequence of moments and the sequence of cumulants, respectively. Let $M(z)=\sum_{k=0}^{\infty} m_{k} z^{k}$ and $C(z)=\sum_{i=0}^{\infty} k_{i} z^{i}$. 
Then, by the definition of the R-transform

$$
\begin{aligned}
R(z) & =\sum_{i=0}^{\infty} k_{i+1} z^{i}=\frac{1}{z} \sum_{i=0}^{\infty} k_{i+1} z^{i+1}=\frac{1}{z} \sum_{i=1}^{\infty} k_{i} z^{i}=\frac{1}{z}\left(\sum_{i=0}^{\infty} k_{i} z^{i}-1\right) \\
& =\frac{1}{z}(C(z)-1)
\end{aligned}
$$

holds. Thus according to Lemma 3.2.1, we get the relation between the moment and cumulant generating functions:

$$
M(z)=C(z M(z)) .
$$

Then

$$
\begin{aligned}
R(G(z))+\frac{1}{G(z)} \stackrel{\stackrel{(3.2)}{=}}{=} \frac{1}{G(z)}(C(G(z))-1)+\frac{1}{G(z)}=\frac{1}{G(z)} C(G(z)) \\
\stackrel{(3.1)}{=} \frac{1}{G(z)} C\left(\frac{1}{z} M\left(\frac{1}{z}\right)\right) \stackrel{(3.3)}{=} \frac{1}{G(z)} M\left(\frac{1}{z}\right) \stackrel{(3.1)}{=} z
\end{aligned}
$$

and the theorem is proved.

Since the R-transform will play an important role in Section 3.3 and in Paper I we prove here some of its properties. The first two in Theorem 3.2 .2 will be of particular importance to us. Note, that the freeness of random variables is essential in part b) of the below given theorem.

Theorem 3.2.2. Let $(\mathcal{A}, \tau)$ be a non-commutative probability space, such that the distributions of $X, Y, X_{n} \in \mathcal{A}$, for all $n \in \mathbb{N}$, have compact support. The $R$-transform satisfies the following properties

a) Non-linearity: $R_{\alpha X}(z)=\alpha R_{X}(\alpha z)$ for every $X \in \mathcal{A}$ and $\alpha \in \mathbb{C}$;

b) For any two free non-commutative random variables $X, Y \in \mathcal{A}$

$$
R_{X+Y}(z)=R_{X}(z)+R_{Y}(z)
$$

c) Let $X, X_{n} \in \mathcal{A}$, for $n \in \mathbb{N}$. If

$$
\lim _{n \rightarrow \infty} \tau\left(X_{n}^{k}\right)=\tau\left(X^{k}\right), \quad k=1,2, \ldots,
$$

and there exists neighborhood $U$ of 0 such that $R_{X}$ and $R_{X_{n}}$ are welldefined for $n \in \mathbb{N}$, then

$$
\lim _{n \rightarrow \infty} R_{X_{n}}(y)=R_{X}(y)
$$

for all $y \in U$ as a formal power series (convergence of coefficients). Note that according to Definition 2.1.4 $\tau\left(X^{k}\right)$ is the kth free moment of the element $X$. 
Proof. The proofs presented below directly follow from the introduced definitions and can be found, for example, in the lecture notes by Hiai (2006).

a) Let us prove the lack of linearity for the R-transform. We first notice that

$$
\begin{aligned}
G_{\alpha X}(z) & =\int_{\mathbb{R}} \frac{1}{z-\alpha x} d \mu(\alpha x)=\int_{\mathbb{R}} \frac{1}{z-\alpha x} d \mu(x)=\frac{1}{\alpha} \int_{\mathbb{R}} \frac{1}{\frac{z}{\alpha}-x} d \mu(x) \\
& =\frac{1}{\alpha} G_{X}\left(\frac{z}{\alpha}\right)
\end{aligned}
$$

and then as $G_{\alpha X}^{-1}\left(G_{\alpha X}(z)\right)=z$ we have

$$
\begin{aligned}
z & =G_{\alpha X}\left(G_{\alpha X}^{-1}(z)\right)=\frac{1}{\alpha} G_{X}\left(\frac{1}{\alpha} G_{\alpha X}^{-1}(z)\right), \\
\alpha z & =G_{X}\left(\frac{1}{\alpha} G_{\alpha X}^{-1}(z)\right), \\
G_{X}^{-1}(\alpha z) & =\frac{1}{\alpha} G_{\alpha X}^{-1}(z),
\end{aligned}
$$

Hence, $G_{\alpha X}^{-1}(z)=\alpha G_{X}^{-1}(\alpha z)$. Then,

$$
\begin{aligned}
R_{\alpha X}(z) & =G_{\alpha X}^{-1}(z)-\frac{1}{z}=\alpha G_{X}^{-1}(\alpha z)-\frac{1}{z}=\alpha\left(G_{X}^{-1}(\alpha z)-\frac{1}{\alpha z}\right) \\
& =\alpha R_{X}(\alpha z) .
\end{aligned}
$$

b) By the freeness of $X$ and $Y$ we have $k_{i}^{X+Y}=k_{i}^{X}+k_{i}^{Y}$ for $i=1,2, \ldots$, see Theorem 2.2.2. Then,

$$
\begin{aligned}
R_{X+Y}(z) & =\sum_{i=0}^{\infty} k_{i+1}^{X+Y} z^{i}=\sum_{i=0}^{\infty}\left(k_{i+1}^{X}+k_{i+1}^{Y}\right) z^{i}=\sum_{i=0}^{\infty} k_{i+1}^{X} z^{i}+\sum_{i=0}^{\infty} k_{i+1}^{Y} z^{i} \\
& =R_{X}(z)+R_{Y}(z)
\end{aligned}
$$

c) The last property follows directly from the definition of the $\mathrm{R}$-transform and the fact that, in neighborhood $U$ of $0, R_{X}$ and $R_{X_{n}}$ are well-defined for $n \in \mathbb{N}$. As the free cumulants converge, the $\mathrm{R}$-transform also converges in each of its coefficients.

Besides the asymptotic freeness of matrices, results regarding the $\mathrm{R}$-transform, Part b) of Theorem 3.2.2 and Theorem 3.2.1, are considered to be the two main achievements presented by Voiculescu in his early paper (Voiculescu, 1991). 


\subsection{Use of Stieltjes and $\mathrm{R}$-transforms for deriv- ing spectral distribution}

In a non-commutative probability space the so-called Wigner semicircle law plays the same role as the Gaussian distribution in classical probability, for example as a limiting distribution in the free version of the Central Limit Theorem.

Definition 3.3.1. Let $X$ be a Hermitian random matrix of size $p \times p$ with entries being i.i.d. Gaussian random variables with mean 0 and variance $1 / p$. Then, the empirical spectral distribution of $X$ converges to the Wigner semicircle law with density function given by

$$
\frac{1}{2 \pi} \sqrt{4-x^{2}} \mathbb{1}_{\{x: x \in(-2,2)\}}, \quad \text { as } \quad p \rightarrow \infty .
$$

This well-known result can be proven using the method based on the Stieltjes transform. That method has been suggested, among others, by Marčenko and Pastur (1967) who used the transform and the inverse Stieltjes transform formula to obtain a result stated in Theorem 3.3.1 below.

In this section we discuss the results that are an illustration of the use of the Stieltjes transform in Random matrix theory. The theorems show various methods of calculations of the asymptotic spectral distribution of $\frac{1}{n} X X^{\prime}$, where $X \sim N_{p, n}\left(0, \sigma^{2} I_{p}, I_{n}\right)$, and the sum of such quadratic forms. Note that $X \sim N_{p, n}(0, \Sigma, \Psi)$ stands for $X$ following the matrix normal distribution. The mean of the matrix normal distribution is a $p \times p$ zero matrix and the dispersion matrix of $X$ has the Kronecker product structure, i.e., $D[X]=D[\operatorname{vec} X]=\Psi \otimes \Sigma$, where both $\Sigma$ and $\Psi$ are positive definite matrices. The notation vec $X$ denotes vectorization of the matrix starting with the first column. If some elements are standardized one can interpret $\Sigma$ and $\Psi$ as the covariance matrices for the rows and the columns of $X$, respectively.

We recall some of the results obtained in Marčenko and Pastur (1967), Girko and von Rosen (1994) and Silverstein and Bai (1995).

Theorem 3.3.1 (Marčenko-Pastur Law). Consider the matrix $\frac{1}{n} X X^{\prime}$, where $X \sim N_{p, n}\left(0, \sigma^{2} I_{p}, I_{n}\right)$. Then the asymptotic spectral distribution is given by: If $\frac{p}{n} \rightarrow c \in(0,1]$

$$
\mu^{\prime}(x)=\frac{\sqrt{\left[\sigma^{2}(1+\sqrt{c})^{2}-x\right]\left[x-\sigma^{2}(1-\sqrt{c})^{2}\right]}}{2 \pi c \sigma^{2} x} \mathbb{1}_{\left((1-\sqrt{c})^{2} \sigma^{2},(1+\sqrt{c})^{2} \sigma^{2}\right)}(x)
$$

If $\frac{p}{n} \rightarrow c \geq 1$

$$
\left(1-\frac{1}{c}\right) \delta_{0}+\mu
$$

where the asymptotic spectral density function $\mu(x)$ follows given above case $c \in(0,1]$. 
In the special case when $c=1$ we obtain the spectral density

$$
\mu^{\prime}(x)=\frac{1}{2 \pi x} \sqrt{4 x-x^{2}},
$$

which is a scaled $\beta$-distribution with parameters $\alpha=\frac{1}{2}$ and $\beta=\frac{3}{2}$.

Moreover, it has been proven that for a class of random matrices with dependent entries, the limiting empirical distribution of the eigenvalues is given by the Marčenko-Pastur law.

Theorem 3.3.2 (Girko and von Rosen 1994). Let $X \sim N_{n, p}(0, \Sigma, \Psi)$, where the eigenvalues of $\Sigma$ and $\Psi$ are bounded by some constant. Suppose that the Kolmogorov condition $0<c=\lim _{n \rightarrow \infty} \frac{p}{n}<\infty$ holds and let $F_{p}^{A A^{\prime}+\frac{1}{n} X X^{\prime}}(x)$ be defined by (2.6), where $A$ is a non-random matrix. Then, for every $x \geq 0$,

$$
F_{p}^{A A^{\prime}+\frac{1}{n} X X^{\prime}}(x)-F_{n}(x) \stackrel{p}{\rightarrow} 0, \quad n \rightarrow \infty,
$$

where $\stackrel{p}{\rightarrow}$ denotes convergence in probability and where for large $n,\left\{F_{n}(x)\right\}$ are distribution functions satisfying

$$
\int_{0}^{\infty} \frac{d F_{n}(x)}{1+t x}=\frac{1}{p} \operatorname{Tr}\left(I+t A A^{\prime}+t \Sigma a(t)\right)^{-1},
$$

where for all $t>0, a(t)$ is a unique non-negative analytical function which exists and which satisfies the nonlinear equation

$$
a(t)=\frac{1}{n} \operatorname{Tr}\left(\Psi\left(I+\frac{t}{n} \Psi \operatorname{Tr}\left(\Sigma\left(I+t A A^{\prime}+t \Sigma a(t)\right)^{-1}\right)\right)^{-1}\right) .
$$

Note, that the Stieltjes transform $G(z)$, defined according to Definition 3.1.1, is given by $G(z)=\frac{1}{z} g\left(-\frac{1}{z}\right)$, where $g(z)=\int_{0}^{\infty} \frac{d F_{n}(x)}{1+z x}$ as in Theorem 3.3.2.

Theorem 3.3.3 (Girko and von Rosen 1994). Consider

$$
\frac{1}{n_{1}} X_{1} X_{1}^{\prime}+\frac{1}{n_{2}} X_{2} X_{2}^{\prime}
$$

where the matrices $X_{1}$ and $X_{2}$ are independent and $X_{i} \sim N_{n, p}\left(0, \Sigma_{i}, \Psi_{i}\right), i=$ 1,2. Let

$$
\begin{aligned}
a(t) & =\frac{1}{n_{2}} \operatorname{Tr}\left(\Psi_{2}\left(I+\frac{t}{n_{2}} \Psi_{2} b(t)\right)^{-1}\right) \\
b(t) & =\frac{1}{n_{2}} \operatorname{Tr}\left(\Sigma_{2}\left(I+t \Sigma_{2} a(t)+t \Sigma_{1} c(t)\right)^{-1}\right) \\
c(t) & =\frac{1}{n_{1}} \operatorname{Tr}\left(\Psi_{1}\left(I+\frac{t}{n_{1}} \Psi_{1} \operatorname{Tr}\left(\Sigma_{1}\left(I+t \Sigma_{2} a(t)+t \Sigma_{1} b(t)\right)^{-1}\right)\right)^{-1}\right), \\
d(t) & =\frac{1}{n_{1}} \operatorname{Tr}\left(\Psi_{1}\left(I+\frac{t}{n_{1}} \Psi_{1} \operatorname{Tr}\left(\Sigma_{1}\left(I+t \Sigma_{2} a(t)+t \Sigma_{1} d(t)\right)^{-1}\right)\right)^{-1}\right) .
\end{aligned}
$$


Put $g(t)=\frac{1}{p} \operatorname{Tr}\left(\left(I+t / n_{1} X_{1} X_{1}^{\prime}+t / n_{2} X_{2} X_{2}^{\prime}\right)^{-1}\right)$. If $0<\lim _{n_{1} \rightarrow \infty} \frac{p}{n_{1}}<\infty$ and $0<\lim _{n_{2} \rightarrow \infty} \frac{p}{n_{2}}<\infty$ it follows that

$$
g(t) \rightarrow \frac{1}{p}\left(I+t \Sigma_{1} d(t)+t \Sigma_{2} a(t)\right)^{-1}, \quad n \rightarrow \infty .
$$

The previously stated theorems of this section assume that the matrix $X$ is real. We present now an example of a similar theorem for a complex random matrix $X$.

Theorem 3.3.4 (Silverstein and Bai 1995). Assume that

- $Z=\left(\frac{1}{\sqrt{p}} Z_{i j}\right)_{p \times n}, Z_{i j} \in \mathbb{C}$, i.i.d. with $\mathbb{E}\left|Z_{11}-\mathbb{E} Z_{11}\right|^{2}=1$, where $|\cdot|$ stands for the absolute value;

- $0<\lim _{n_{1} \rightarrow \infty} \frac{p}{n}=c<\infty$ as $p, n \rightarrow \infty$ (Kolmogorov condition);

- $T=\operatorname{diag}\left(\tau_{1}, \tau_{2}, \ldots, \tau_{n}\right)$ where $\tau_{i} \in \mathbb{R}$, and the empirical distribution function of the eigenvalues of the matrix T, i.e., $\left\{\tau_{1}, \tau_{2}, \ldots, \tau_{n}\right\}$, converges almost surely in distribution to a probability distribution function $H$ as $p \rightarrow \infty$ (hence as $n(p)$ increases);

- $B=A+Z T Z^{*}$, where $A$ is a Hermitian $p \times p$ matrix for which $F^{A}$ converges vaguely to $\nu$, where $\nu$ is a possibly defective (i.e., with discontinuities) distribution function, i.e., there exists an everywhere dense subset $D$ of $\mathbb{R}$ such that

$$
\forall_{a, b \in D, a<b} \quad F^{A}(a, b] \rightarrow \nu(a, b], \quad n, p \rightarrow \infty .
$$

- $Z, T$ and $A$ are independent.

Then $F^{B}$, the empirical distribution function of the eigenvalues of $B$, converges almost surely, as $p, n \rightarrow \infty$ to a distribution function $F$ whose Stieltjes transform equals $m(z), z \in \mathbb{C}^{+}$and satisfies the canonical equation

$$
m(z)=m_{\nu}\left(z-\frac{1}{c} \int \frac{\tau d H(\tau)}{1+\tau m(z)}\right) .
$$

Note that Silverstein and Bai defined the Stieltjes transform as $-G(z)$, where $G(z)$ is given by Definition 3.1.1. Hence, the inverse Stieltjes transform is also given with the opposite sign, i.e.,

$$
\mu(I)=\frac{1}{\pi} \lim _{y \rightarrow 0} \int_{I} \Im G(x+\mathrm{i} y) d x
$$

where $I=(a, b)$ such that $a, b$ are not atoms for the measure $\mu$. 
Theorem 3.3.2 together with Theorem 3.3.4 provide us with two computationally different ways to obtain the asymptotic spectral distribution. The aforementioned theorems give us the Stieltjes transforms, which, however, differ by a vanishing term, and therefore lead to the same asymptotic distribution function. To illustrate those differences with a simple example based on $\frac{1}{n} X X^{\prime}$, where $X \sim N_{p, n}\left(0, \sigma^{2} I, I\right)$ we refer to Pielaszkiewicz (2013).

Due to the obtained asymptotic spectral distribution of $Q_{n}=\frac{1}{n} \sum_{i=1}^{k} X_{i} X_{i}^{\prime}$, where $X_{i}$ are independent normally distributed matrices, asymptotic free independence of the sum of the elements $X_{i} X_{i}^{\prime}$ can be used. Then the sum of the $\mathrm{R}$-transforms for asymptotically free independent elements leads us to the R-transform of $Q_{n}$. The difficulty here is to be able to analytically calculate the inverse Stieltjes transform. The general idea of conducting calculations is given by Figure 3.1 and is used in Paper I.

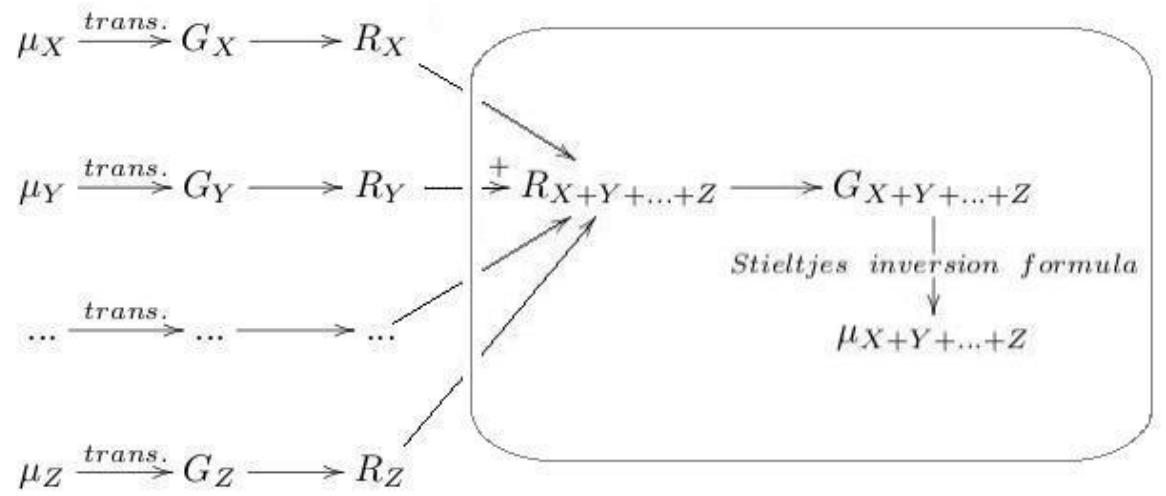

Figure 3.1: Graphical illustration of the procedure of calculating the asymptotic spectral distribution function using the knowledge about asymptotic spectral distribution of its asymptotically free independent summands. By "trans." we indicate step of calculating the Stieltjes transform $G_{\bullet}$. The steps must be asymptotic latest in the surrounded area due to the requirement of freeness.

Following the arrows in Figure 3.1 we recall that the distribution of each of the asymptotically free independent summands leads to the corresponding Stieltjes transform. Then, using Theorem 3.2.1 we obtain the $\mathrm{R}$-transforms, which are later added. The form of the calculated Stieltjes transform $G_{X+Y+\ldots+Z}$ allows us, in some cases, to obtain a closed form expression for the asymptotic spectral density function. A particular class of matrices with a closed form solution is given by Theorem 3.3.5. In applications to areas such as signal processing, the problem of the lack of a closed form expression for the asymptotic spectral distribution is solved numerically, see Chen et al. (2012) and Paper I. 
Theorem 3.3.5. Let $Q_{n}$ be a matrix of size $p \times p$ defined as

$$
Q_{n}=\frac{1}{n} X_{1} X_{1}^{\prime}+\cdots+\frac{1}{n} X_{k} X_{k}^{\prime}
$$

where $X_{i} \sim \mathcal{N}_{p, n}\left(0, \sigma^{2} I_{p}, I_{n}\right)$, i.e., $X_{i}$ is a $p \times n$ matrix following a matrix normal distribution. Then, the asymptotic spectral distribution of $Q_{n}$, denoted by $\mu$, is determined by the spectral density function

$$
\mu^{\prime}(x)=\frac{\sqrt{\left[\sigma^{2}(\sqrt{k}+\sqrt{c})^{2}-x\right]\left[x-\sigma^{2}(\sqrt{k}-\sqrt{c})^{2}\right]}}{2 \pi c x \sigma^{2}} \mathbb{1}_{M}(x),
$$

where $M=\left(\sigma^{2}(\sqrt{k}-\sqrt{c})^{2}, \sigma^{2}(\sqrt{k}+\sqrt{c})^{2}\right), n \rightarrow \infty$ and the Kolmogorov condition, $\lim _{n \rightarrow \infty} \frac{p(n)}{n}=c$, holds.

Following Paper I, classes of matrices for which it is possible to obtain a closed form of the asymptotic spectral distribution are characterized by a general form of the inverse with respect to the composition of the Stieltjes transform, as in the following theorem.

Theorem 3.3.6 (Paper I). For any $p \times p$ dimensional matrix $Q \in \mathcal{Q}$ whose inverse, with respect to the composition, of Stieltjes transform is of the form

$$
G^{-1}(z)=\frac{a z+b}{c z^{2}+d z+e}
$$

where $a, b, c, d, e \in \mathbb{R}, c \neq 0, d^{2}-4 c e \neq 0$ the asymptotic spectral distribution is given by

$$
\mu^{\prime}(x)=\frac{1}{2 \pi x} \sqrt{\left(-d^{2}+4 c e\right) x^{2}-a^{2}-2(2 b c-a d) x} \mathbb{1}_{D}(x),
$$

when the Kolmogorov condition holds, i.e., $\frac{p(n)}{n} \rightarrow c \in(0, \infty)$ for $n \rightarrow \infty$ and $D:=\left\{x: \frac{a d-2 b c-2 \sqrt{b^{2} c^{2}-a b c d+a^{2} c e}}{d^{2}-4 c e}<x<\frac{a d-2 b c+2 \sqrt{b^{2} c^{2}-a b c d+a^{2} c e}}{d^{2}-4 c e}\right\}$.

Remark 3.3.1. The class of matrices whose inverse, with respect to the composition, of the Stieltjes transform, given by Theorem 3.3.6, is identical to the class of matrices with $R$-transform given by:

$$
R(z)=\frac{a z+b}{c z^{2}+d z+e}-\frac{1}{z}=\frac{(a-c) z^{2}+(b-d) z-e}{z\left(c z^{2}+d z+e\right)} .
$$





\section{Wishart matrices}

Let the matrix $X$ be of size $p \times n$, then the $p \times p$ matrix $X X^{\prime}$ can be considered as an element of the non-commutative algebra of all $p \times p$ matrices, as discussed in Chapter 2. Furthermore, in Section 3.3 of Chapter 3, we have discussed a number of results regarding the spectral distribution of matrices of type $\frac{1}{n} X X^{\prime}$, where $X \sim N_{p, n}\left(0, \sigma^{2} I_{p}, I_{n}\right)$. In this chapter we would like to focus on $W=X X^{\prime}$ itself, where $X \sim N_{p, n}\left(M, \Sigma, I_{n}\right)$, i.e., $W$ is a Wishart matrix. Wishart matrices are commonly used in statistics. For example, in multivariate statistics under a normality assumption, the distribution of the sample-covariance matrix is Wishart distributed. Moreover, many test statistics considered in classical multivariate analysis are given as a function of one or several Wishart matrices. In this chapter we will give a definition of the Wishart distribution together with a number of related properties, as well as comment on the statistical use of such objects, in particular put some results connected to eigenvalues of Wishart matrices. For example, the expectation of the product of traces of Wishart matrices is studied. These quantities can be used when approximating densities. Kawasaki and Seo (2012) considered tests for mean vectors with unequal covariance matrices and then used moments of trace products of Wishart matrices. Moreover, when expanding the Stieltjes transform, the moments of products of traces appear (see Section 3.1).

\subsection{Wishart distribution}

Following Timm (2007), Mukhopadhyay (2009) and Kollo and von Rosen (2005) we start with a definition of the Wishart distribution.

Definition 4.1.1. The matrix $W$ of size $p \times p$ is said to be Wishart distributed if and only if $W=X X^{\prime}$ for some matrix $X$, where $X \sim \mathcal{N}_{p, n}\left(M, \Sigma, I_{n}\right)$ and the matrix $\Sigma \geq 0$.

In particular, we speak about a central Wishart distribution if $M=0$, which is denoted $W \sim \mathcal{W}_{p}(\Sigma, n)$.

In the case when $X$ is not centered, i.e., $X \sim \mathcal{N}_{p, n}\left(M, \Sigma, I_{n}\right)$, where $M \neq 0$, we talk about a non-central Wishart distribution characterized by the parameter $n$, $\Sigma$ and $\Delta=M M^{\prime}$, and denoted $X X^{\prime} \sim \mathcal{W}_{p}(\Sigma, n, \Delta)$. 
The probability density function of $W \sim \mathcal{W}_{p}(\Sigma, n)$, for $n \geq p$ and positive definite matrix $\Sigma$, is given by

$$
f(W)=\left\{2^{\frac{n p}{2}} \Gamma_{p}\left(\frac{n}{2}\right)|\Sigma|^{\frac{n}{2}}\right\}^{-1}|W|^{\frac{1}{2}(n-p-1)} e^{-\frac{1}{2} \operatorname{Tr}\left\{\Sigma^{-1} W\right\}}, \quad \text { for } W>0,
$$

where $|\Sigma|$ stands for a determinant of the matrix $\Sigma, W>0$ denotes positive definiteness of the matrix $W$ and the multivariate gamma function is given by

$$
\Gamma_{p}\left(\frac{n}{2}\right)=\pi^{\frac{p(p-1)}{4}} \prod_{i=1}^{p} \Gamma\left(\frac{n+1-i}{2}\right) .
$$

The density function is given for the positive definite matrix $W$, i.e., $W>0$, since in the case when $W \geq 0$ we have $P(|W|=0)=1$ and then the density function (4.1) does not exist.

The Wishart distribution is a generalization of the univariate $\chi^{2}$-distribution and shares a number of properties with that distribution. The distribution was derived in the early 20's by Fisher (1915) for $p=2$ while he was studing the distribution of correlation coefficients of a normal sample. Generalization to an arbitrary $p$ was given in Wishart (1928). Wigner (1958) studied the spectral distribution related to heavy-nuclei atoms. For more information, detailed proofs and applications to other fields such as wireless communication, see the books Gupta and Nagar (1999), Tulino and Verdu (2004), Girko (1990), Mehta (1991), Muirhead (1982) and Anderson (2003).

The density function of a non-central Wishart distribution is a generalization of the one presented for the centered case and it can be found in Muirhead (1982).

Some authors, e.g., Mardia et al. (1979), Gupta and Nagar (1999), define the Wishart distribution through its distribution function. A proof that the random variable with density function given by (4.1) can be decomposed as $W=X X^{\prime}$, as given in Definition 4.1.1, is presented, in the case of integer $p$, in the book by Gupta and Nagar (1999). In high-dimensional cases, i.e., $p>n$, the distribution is called singular Wishart (see Mukhopadhyay, 2009; Seber, 2009), anti-Wishart (see article by Janik and Nowak, 2003) or pseudo-Wishart (see, Fujikoshi et al., 2011).

Furthermore, the characteristic functions of a central Wishart and non-central Wishart distribution are given by the following theorem (see Muirhead, 1982).

Theorem 4.1.1. Let $W_{1}$ be a non-central Wishart matrix denoted by $W_{1} \sim$ $\mathcal{W}_{p}(\Sigma, n, \Delta)$ and $W_{2}$ be a central Wishart matrix, i.e., $W_{2} \sim \mathcal{W}_{p}(\Sigma, n)$. Then, the characteristic functions of $W_{1}$ and $W_{2}$ (joint characteristic function of the $\frac{p(p+1)}{2}$ upper triangular matrix elements) are given as

$$
\begin{aligned}
& \phi_{W_{1}}(T)=\left|I_{p}-2 i \Sigma Z\right|^{-\frac{n}{2}} e^{\operatorname{Tr}\left\{-\frac{1}{2} \Delta+\frac{1}{2}\left(I_{p}-2 i \Sigma Z\right)^{-1} \Delta\right\}}, \\
& \phi_{W_{2}}(T)=\left|I_{p}-2 i \Sigma Z\right|^{-\frac{n}{2}},
\end{aligned}
$$


respectively, where the square matrix $Z$ of size $p \times p$ equals $Z=\left(\frac{1}{2}\left(1+\delta_{i j}\right) t_{i j}\right)_{i, j=1}^{p}$ with the symmetric matrix $T=T^{\prime}=\left(t_{i j}\right)_{i, j=1}^{p}$ and $\delta_{i j}$ standing for the Kronecker delta.

The Wishart distribution can also be defined through the characteristic function, which allows for the non-integer $n$. As mentioned earlier, the Wishart distribution is a generalization of the $\chi^{2}$ distribution. Indeed, putting $p=1$ (and $\Sigma=1$ ) implies that $W$ is a random variable and its density function (4.1), if it exists, equals

$$
\left\{2^{\frac{n}{2}} \Gamma\left(\frac{n}{2}\right)\right\}^{-1} W^{\frac{1}{2}(n-2)} e^{-\frac{1}{2} W},
$$

which is the density function of a $\chi^{2}$-distributed random variable with $n$ degrees of freedom.

For future use, in comparisons contained in Section 4.3.3 and Paper III, we shortly define the complex Wishart distribution. An early reference here is Goodman (1963) and an alternative definition of the complex Wishart distribution is based on the Laplace transform, see Graczyk et al. (2003), Bryc (2008a).

Let the complex $p$-dimensional column vector $X_{i}$ follow the complex normal distribution, i.e., $X_{i} \sim \mathcal{N}_{p}^{\mathbb{C}}(\mu, \Sigma)$. That is equivalent to saying that the corresponding $2 p$-dimensional vector $Y_{i}=\left(\Re X_{i}^{\prime}, \Im X_{i}^{\prime}\right)^{\prime} \sim \mathcal{N}_{2 p}\left(\left(\Re \mu^{\prime}, \Im \mu^{\prime}\right)^{\prime}, \Sigma_{Y_{i}}\right)$, where

$$
\Sigma_{Y_{i}}=\frac{1}{2}\left(\begin{array}{cc}
\Re \Sigma & -\Im \Sigma \\
\Im \Sigma & \Re \Sigma
\end{array}\right),
$$

$\Im X_{i}$ and $\Re X_{i}$ denote the imaginary and real part, respectively, of the complex $X_{i}$ and where the matrix $\Im \Sigma$ is skew-symmetric.

Again let $X=\left(X_{1}, \ldots, X_{n}\right)$ where $X_{i} \sim \mathcal{N}_{p}^{\mathbb{C}}(\mu, \Sigma)$ which are supposed to be independently distributed for $i=1, \ldots, n$. Then a matrix $W_{\mathbb{C}} \sim \mathcal{W}_{p}^{\mathbb{C}}(\Sigma, n)$ is complex Wishart distributed if $W_{\mathbb{C}}=X X^{*}$, where ${ }^{*}$ denotes the conjugate transpose.

\subsection{Properties of Wishart matrices}

In this section we will give a number of basic properties of Wishart matrices that are used in the thesis or are closely related to the content.

Theorem 4.2.1. Let $X \sim \mathcal{N}_{p, n}(M, \Sigma, \Omega)$ and $A$ be a symmetric matrix of size $n \times n$. Then, $X A X^{\prime} \sim \mathcal{W}_{p}\left(\Sigma, q, M A M^{\prime}\right)$ if and only if $A \Omega$ is idempotent, i.e., $A \Omega=A \Omega A \Omega$, where the rank of matrix $A \Omega$ equals $q$.

Corollary 4.2.1. Let $X \sim \mathcal{N}_{p, n}\left(0, \Sigma, I_{n}\right)$ and $A$ be a symmetric and idempotent matrix of size $n \times n$, i.e., $A=A A$, of rank $q>p$. Then, $X A X^{\prime} \sim \mathcal{W}_{p}(\Sigma, q)$. 
Consider $X \sim \mathcal{N}_{p, n}\left(\mu \mathbf{1}_{p}^{\prime}, \Sigma, I_{n}\right)$, where $\mu=\left(\mu_{1}, \ldots, \mu_{p}\right)^{\prime}$ (so $X$ consists of $n$ independent vectors of length $\left.p, X=\left(X_{1}, \ldots, X_{n}\right)\right)$ and the matrix $C=I_{n}-$ $\frac{1}{n} \mathbf{1 1}$. The chosen matrix $C$ is of rank $n-1$, idempotent and symmetric. Then, using the above theorem we have

$$
X C X^{\prime} \sim \mathcal{W}_{p}(\Sigma, n-1) .
$$

Note that with this $C$, we have $X C X^{\prime}=X\left(I_{n}-\frac{1}{n} \mathbf{1} \mathbf{1}^{\prime}\right) X^{\prime}=\left(X-\bar{x} \mathbf{1}_{p}\right)^{\prime}(X-$ $\left.\bar{x} \mathbf{1}_{p}\right)=n S$, where $S$ stands for the sample covariance matrix and $\bar{x}=\frac{1}{n} \sum_{i=1}^{n} X_{i}$. Moreover, it can be shown that the sample covariance matrix $S$ and mean vector $\bar{x}$ are independent. Expression $\frac{1}{n-1} X C X^{\prime}$ is often considered, because it is an unbiased estimator of $\Sigma$.

Theorem 4.2.2. Let $X \sim \mathcal{N}_{p, n}\left(0, \Sigma, I_{n}\right)$ and $A$ be a symmetric positive semidefinite matrix of size $p \times p$ and of rank $q \geq n$ such that $A \Sigma A=A$. Then, $X^{\prime} A X \sim \mathcal{W}_{n}\left(I_{n}, q\right)$.

Theorem 4.2.3. Let $W \sim \mathcal{W}_{p}(\Sigma, n)$ and $A$ be a matrix of size $q \times p$. Then, $A W A^{\prime} \sim \mathcal{W}_{q}\left(A \Sigma A^{\prime}, n\right)$.

Hence, $\Sigma^{-\frac{1}{2}} W \Sigma^{-\frac{1}{2}} \sim \mathcal{W}_{p}\left(I_{p}, n\right)$, if $W \sim \mathcal{W}_{p}(\Sigma, n)$.

Moreover, as we are considering sums of Wishart matrices in Paper I, among others, it is good to recall the following theorem.

Theorem 4.2.4. Let $W_{1} \sim \mathcal{W}_{p}\left(\Sigma, n_{1}\right)$ and $W_{2} \sim \mathcal{W}_{p}\left(\Sigma, n_{2}\right)$ be independent. Then, $W_{1}+W_{2} \sim \mathcal{W}_{p}\left(\Sigma, n_{1}+n_{2}\right)$.

\subsection{On $\prod_{i=0}^{k} \operatorname{Tr}\left\{W^{m_{i}}\right\}$}

It is of interest to consider

$$
\mathbb{E}\left[\prod_{i=0}^{k} \operatorname{Tr}\left\{W^{m_{i}}\right\}\right], \quad m_{i} \geq 0, \quad i=0, \ldots, k,
$$

where $W \sim \mathcal{W}_{p}\left(I_{p}, n\right)$ since its version appears in the application of the free cumulant-moment formula for deriving the moments of the Marčenko-Pastur distribution discussed in Paper II. Moreover according to Capitaine and Casalis (2004) $\mathbb{E}\left[\prod_{i=0}^{k} \operatorname{Tr}\left\{W^{m_{i}}\right\}\right]$ can be seen as a special case of generalized moments. The study is carried out in Paper III.

The special case $\mathbb{E}\left[\operatorname{Tr}\left\{W^{l}\right\}\right]$ coincides with the free moments of the empirical spectral distribution for $W$ (see, Subsection 2.1.2). Asymptotically $\left(\frac{n}{p} \rightarrow c>0\right.$ ) (4.2) gives the moments of the Marčenko-Pastur distribution, see also the original paper by Marčenko and Pastur (1967). Observe that since the MarčenkoPastur distribution is compactly supported, the moments carry the full information about the distribution. 
For both real and complex Wishart matrices, a number of results for (4.2) are available. We focus on the choice of those which, in our opinion, have an applicable form. It is worth pointing out that there exist formulas, for instance Wishart identities, which in a particular case treat the same problem, but demand non trivial differential calculations. For example, see Loh (1991) and Konno (2009) for further reading.

\subsubsection{Real Wishart matrix}

Let $W \sim \mathcal{W}_{p}(\Sigma, n)$. Then, by Theorem 4.2 .3 we have $\Sigma^{-1 / 2} W \Sigma^{-1 / 2} \sim \mathcal{W}_{p}\left(I_{p}, n\right)$. Thus, $E\left[\operatorname{Tr}\left\{\Sigma^{-1} W\right\}\right]$ equals $E[\operatorname{Tr}\{V\}]$, where $V \sim \mathcal{W}_{p}\left(I_{p}, n\right)$.

The expectation of the $k$ th power of the trace of the Wishart matrix, $W \sim$ $\mathcal{W}_{p}(\Sigma, n)$, has been derived by Nel (1971). The general formula involves zonal polynomials and the following Theorem 3.3.23 in Gupta and Nagar (1999) is given by

$$
\mathbb{E}\left[(\operatorname{Tr}\{W\})^{k}\right]=2^{k} \sum_{\kappa}\left[\frac{n}{2}\right]_{\kappa} Z_{\kappa}(\Sigma),
$$

where $Z_{\kappa}(\cdot)$ stands for the zonal polynomial corresponding to $\kappa$, where $\kappa=$ $\left(k_{1}, \ldots, k_{m}\right)$ is a partition of $k$ such that $k_{i} \geq 0$ for all $i=1, \ldots, m$ and $\sum_{i} k_{i}=$ $k$. We use notation $[a]_{\kappa}=\prod_{i=1}^{m}[a-i+1]_{k_{i}}$ with $[a]_{k}=(a+k) ! / a$ ! for $a \in \mathbb{C}$ and $k \in \mathbb{N}_{0}$. An alternative version of the closed formula for the expectation of the power of the trace of the Wishart matrix $W$ can be found in Mathai (1980).

Using results for zonal polynomials, see e.g., Subrahmaniam (1976),

$$
\mathbb{E}\left[\left(\operatorname{Tr}\left\{\Sigma^{-1} W\right\}\right)^{k}\right]=2^{k}\left[\frac{n p}{2}\right]_{k} .
$$

For any $k \in \mathbb{N}$, a theorem stated in Letac and Massam (2004) gives an alternative formula

$$
\mathbb{E}\left[\left(\operatorname{Tr}\left\{\Sigma^{-1} W\right\}\right)^{k}\right]=\sum_{(i) \in \mathcal{I}_{k}} \frac{k !(n p)^{i_{1}+\ldots+i_{k}}}{i_{1} ! \cdots i_{k} ! 1^{i_{1}} \cdots k^{i_{k}}} 2^{i_{2}+2 i_{3}+\ldots+(k-1) i_{k}},
$$

where the set $\mathcal{I}_{k}$ consists of $k$-tuples $(i)=\left(i_{1}, \ldots, i_{k}\right)$ such that $i_{1}+2 i_{2}+\ldots+$ $k i_{k}=k$ and $i_{j}, j=1, \ldots, k$ are non-negative integers. Moreover, several other results and references are mentioned in Glueck and Muller (1998). In the paper by James (1964), results of the expectation of $\prod_{i=0}^{k} \operatorname{Tr}\left\{W^{m_{i}}\right\}$ are presented with the use of zonal polynomials and representation theory. Zonal polynomials are also explored to obtain the moments of traces of Wishart matrices in Watamori (1990). Furthermore, the recent paper Matsumoto (2012) employs the orthogonal Weingarten function to compute general moments of the real Wishart matrix as well as of its inverse.

Above, the expectation of the power of the trace of a real central Wishart matrix has been presented. Other formulations are about the expectation of the trace 
of the power, i.e., $\mathbb{E}\left[\operatorname{Tr}\left\{\left(\Sigma^{-1} W\right)^{l}\right\}\right]$. For a non-central Wishart distribution, $V \sim$ $\mathcal{W}_{p}(\Sigma, n, \Delta)$, i.e., a Wishart matrix which is defined via $X \sim \mathcal{N}_{p, n}\left(M, \Sigma, I_{n}\right)$ as $V=X X^{\prime}$ with $\Delta=M M^{\prime}$, it is well known that the following holds (see Gupta and Nadarajah, 2004, for more details).

$$
\begin{aligned}
\mathbb{E}\left[\operatorname{Tr}\left\{V \Sigma^{-1}\right\}\right] & =n p+\operatorname{Tr}\{\Delta\}, \\
\mathbb{E}\left[\operatorname{Tr}\left\{\left(V \Sigma^{-1}\right)^{2}\right\}\right] & =n p(n+p+1)+2(n+p+1) \operatorname{Tr}\{\Delta\}+\operatorname{Tr}\left\{\Delta^{2}\right\} .
\end{aligned}
$$

For $W \sim \mathcal{W}_{p}(\Sigma, n)$ (see e.g., Fujikoshi et al. (2011))

$$
\mathbb{E}\left[\operatorname{Tr}\left\{W^{2}\right\}\right]=\left(n+n^{2}\right) \operatorname{Tr}\left\{\Sigma^{2}\right\}+n(\operatorname{Tr}\{\Sigma\})^{2} .
$$

Letac and Massam (2008) derived an expression for

$$
\mathbb{E}\left[\prod_{i=0}^{k} \operatorname{Tr}\left\{\left(W H_{i}\right)\right\}\right]
$$

where $H_{1}, \ldots, H_{k}$ are arbitrary real symmetric matrices. Appropriate choice of $H_{i}$ allows to obtain moments of all monomials. Using umbral calculations, i.e., a symbolic method introduced by Rota and Taylor (1994), a generalization of (4.6) to a non-central Wishart matrix has been suggested by Nardo (2014).

In Paper III we derived the following recursive formula for $W \sim \mathcal{W}_{p}\left(I_{p}, n\right)$.

Theorem 4.3.1. Let $W \sim \mathcal{W}_{p}\left(I_{p}, n\right)$. Then, the following recursive formula holds for all $k \in \mathbb{N}$ and all $m_{0}, m_{1}, \ldots, m_{k}$ such that $m_{0}=0, m_{k} \in \mathbb{N}, m_{i} \in \mathbb{N}_{0}$, $i=1, \ldots, k-1$

$$
\begin{aligned}
\mathbb{E}\left[\prod_{i=0}^{k} \operatorname{Tr}\left\{W^{m_{i}}\right\}\right]= & \left(n-p+m_{k}-1\right) \mathbb{E}\left[\operatorname{Tr}\left\{W^{m_{k}-1}\right\} \prod_{i=0}^{k-1} \operatorname{Tr}\left\{W^{m_{i}}\right\}\right] \\
& +2 \sum_{i=0}^{k-1} m_{i} \mathbb{E}\left[\operatorname{Tr}\left\{W^{m_{k}+m_{i}-1}\right\} \prod_{\substack{j=0 \\
j \neq i}}^{k-1} \operatorname{Tr}\left\{W^{m_{j}}\right\}\right] \\
& +\sum_{i=0}^{m_{k}-1} \mathbb{E}\left[\operatorname{Tr}\left\{W^{i}\right\} \operatorname{Tr}\left\{W^{m_{k}-1-i}\right\} \prod_{j=0}^{k-1} \operatorname{Tr}\left\{W^{m_{j}}\right\}\right] .
\end{aligned}
$$

In particular, the following corollaries can be given.

Corollary 4.3.1. Let $W \sim \mathcal{W}_{p}\left(I_{p}, n\right)$, then for all $l \in \mathbb{N}_{0}$

$\mathbb{E}\left[\operatorname{Tr}\left\{W^{l+1}\right\}\right]=p[n-p-1]_{l+1}+\sum_{i=0}^{l}[n-p+i]_{l-i} \sum_{j=0}^{i} \mathbb{E}\left[\operatorname{Tr}\left\{W^{j}\right\} \operatorname{Tr}\left\{W^{i-j}\right\}\right]$.

Corollary 4.3.2. Let $W \sim \mathcal{W}_{p}\left(I_{p}, n\right)$, then the following recursive formula holds for all $t \in \mathbb{N}$

$$
\mathbb{E}\left[(\operatorname{Tr}\{W\})^{t+1}\right]=(n p+2 t) \mathbb{E}\left[(\operatorname{Tr}\{W\})^{t}\right]=\frac{(n p+2 t) ! !}{(n p) ! !} n p,
$$


where $k !$ ! stands for double factorial of $k$, i.e., $k ! !=k \cdot(k-2) ! !$ for $k \geq 2$, and $k ! !=1$ for $k \in\{0,1\}$.

Its asymptotic version, under the Kolmogorov condition, has been proved in the same paper and is given by

$$
Q^{\mathcal{I}_{(k)}}\left(m_{k}\right)= \begin{cases}\left(\frac{1}{c}+1\right) Q^{\mathcal{I}_{(k)}}\left(m_{k}-1\right)+\frac{1}{c} \sum_{i=1}^{m_{k}-2} Q^{\mathcal{I}_{(k)} \cup\left\{m_{k}-1-i\right\}}(i), & m_{k}>2, k \geq 1, \\ \left(\frac{1}{c}+1\right) Q^{\mathcal{I}_{(k)}}(1), & m_{k}=2, k \geq 1, \\ Q^{\mathcal{I}_{(k)} \backslash\left\{m_{k-1}\right\}}\left(m_{k-1}\right), & m_{k}=1, k>1, \\ 1, & m_{k}=1, k=1 .\end{cases}
$$

where

$$
Q^{\mathcal{I}_{(k)}}\left(m_{k}\right):=\lim _{n, p \rightarrow \infty} \frac{1}{p^{k+1} n^{s}} \mathbb{E}\left[\prod_{i=0}^{k} \operatorname{Tr}\left\{\left(X X^{\prime}\right)^{m_{i}}\right\}\right],
$$

$\mathcal{I}_{(k)}=\left\{m_{0}, \ldots, m_{k-1}\right\}$ is a notation for the $k$-element set of powers of Wishart matrices and $s=\sum_{i=0}^{k} m_{i}$ and $\mathcal{I}_{(k)} \cup\left\{m_{k}-1-i\right\}$ stands for $\left\{m_{0}, \ldots, m_{k-1}, m_{k}-\right.$ $1-i\}$. The formula is recursive with respect to the last power of the Wishart matrix, i.e., $m_{k}$. If $m_{k}>1$ in the next step we decrees $m_{k}$ by one to $m_{k}-1$. If $m_{k}=1, k$ th power vanishes and the next step we use recurrence with respect to $m_{k-1}$. The purpose of the following example is to clarify notation.

Example 4.3.1. Assume that we want to calculate

$$
\lim _{n, p \rightarrow \infty} \mathbb{E}\left[\frac{1}{p} \operatorname{Tr}\left\{\left(\frac{1}{n} X X^{\prime}\right)^{2}\right\} \frac{1}{p} \operatorname{Tr}\left\{\left(\frac{1}{n} X X^{\prime}\right)^{3}\right\}\right],
$$

which after simple transformation equals

$$
Q^{\mathcal{I}_{(2)}}(3)=\lim _{n, p \rightarrow \infty} \frac{1}{p^{3} n^{5}} \mathbb{E}\left[\operatorname{Tr}\left\{\left(X X^{\prime}\right)^{0}\right\} \operatorname{Tr}\left\{\left(X X^{\prime}\right)^{2}\right\} \operatorname{Tr}\left\{\left(X X^{\prime}\right)^{3}\right\}\right],
$$

where $\mathcal{I}_{(2)}=\left\{m_{0}, m_{1}\right\}=\{0,2\}, m_{k}=m_{2}=3$ and $\lim _{p, n \rightarrow \infty} \frac{n}{p}=c \in(0, \infty)$. Then using the formula (4.8) once we obtain

$$
\begin{aligned}
\lim _{n, p \rightarrow \infty} & \mathbb{E}\left[\frac{1}{p} \operatorname{Tr}\left\{\left(\frac{1}{n} X X^{\prime}\right)^{2}\right\} \frac{1}{p} \operatorname{Tr}\left\{\left(\frac{1}{n} X X^{\prime}\right)^{3}\right\}\right] \\
= & \left(\frac{1}{c}+1\right) Q^{\{0,2\}}(2)+\frac{1}{c} \sum_{i=1}^{1} Q^{\{0,2,2-i\}}(i) \\
= & \left(\frac{1}{c}+1\right) Q^{\{0,2\}}(2)+\frac{1}{c} Q^{\{0,2,1\}}(1) .
\end{aligned}
$$

We use the asymptotic result presented above in Subsection 4.3.4 to find the asymptotic distribution of $\frac{1}{p} \operatorname{Tr}\left\{\left(\frac{1}{n} X X^{\prime}\right)^{t}\right\}$. Moreover, formula (4.7) is a tool to investigate the traces of 1st and 2nd order powers of Wishart matrices in Paper IV. Apart from the fact that the formula is quite straightforward to use, the practical advantage is the existence of an implemented function, Paper III, to make recursive calculations for given $n$ and $p$. 


\subsubsection{Complex Wishart matrix}

In this thesis we focus on real Wishart matrices. Despite this fact, there are a number of results derived for complex Wishart matrices, and we would like to show that they can not be utilized in real settings. In this section we give a number of results derived for complex Wishart matrices that will be compared to the results given for the real Wishart version.

Let $W_{\mathbb{C}} \sim \mathcal{W}_{p}^{\mathbb{C}}\left(I_{p}, n\right)$. The matrix $W_{\mathbb{C}}$ is considered in Hanlon et al. (1992), where they found

$$
\mathbb{E}\left[\operatorname{Tr}\left\{W_{\mathbb{C}}^{k}\right\}\right]=\frac{1}{k} \sum_{j=1}^{k}(-1)^{j-1} \frac{[n+k-j]_{k}[p+k-j]_{k}}{(k-j) !(j-1) !}, \quad k \in \mathbb{N} .
$$

A corresponding recursive result was derived by Haagerup and Thorbjornsen (2003), i.e., for all $k \in \mathbb{N}$ the following holds

$$
\begin{aligned}
\mathbb{E}\left[\operatorname{Tr}\left\{W_{\mathbb{C}}^{0}\right\}\right] & =p, \\
\mathbb{E}\left[\operatorname{Tr}\left\{W_{\mathbb{C}}^{1}\right\}\right] & =n p, \\
\mathbb{E}\left[\operatorname{Tr}\left\{W_{\mathbb{C}}^{k+1}\right\}\right] & =\frac{(2 k+1)(n+p)}{k+2} \mathbb{E}\left[\operatorname{Tr}\left\{W_{\mathbb{C}}^{k}\right\}\right]+\frac{(k-1)\left(k^{2}-(n-p)^{2}\right)}{k+2} \mathbb{E}\left[\operatorname{Tr}\left\{W_{\mathbb{C}}^{k-1}\right\}\right] .
\end{aligned}
$$

The given recursive formula (4.10) was inspired by the Harer-Zagier recursion formula. The original paper, Harer and Zagier (1986), gives a recursive relation for even moments of the spectral distribution of a complex self-adjoint random matrix $Z=\left(Z_{i j}\right)$ formed by $p^{2}$ independent real standard normal distributed variables such that $Z_{i j}$ has mean 0 and variance 1 , i.e., an expression for $\frac{1}{p} \mathbb{E}\left[\operatorname{Tr}\left\{Z^{2 k}\right\}\right]$. It has been re-established and extended to the interesting case of Wishart matrices in Haagerup and Thorbjornsen (2003). The proof of (4.10) is mostly combinatorial, while the proof given in Harer and Zagier (1986) combines advanced tools of the mathematical analysis and theory of solving differential equations. Both the explicit result given in Hanlon et al. (1992) and the recursive result of Haagerup and Thorbjornsen (2003) present $\mathbb{E}\left[\operatorname{Tr}\left\{W_{\mathbb{C}}^{k}\right\}\right]$, although equation (4.10) is said by Haagerup and Thorbjornsen (2003) to be more efficient than (4.9) for the generation of moment tables.

\subsubsection{Comparison of results for complex and real Wishart matrices}

Let us illustrate the difference between the real and complex version of results with formulas (4.7) and (4.10). The difference was already claimed in a number of papers. We quote Bryc (2008b): 
Explicit formulas for moments of polynomials in one real Wishart matrix appear in (...). The formulas are more complicated than the formulas for the complex case (...), and often involve sophisticated tools, like wreath products, Jack polynomials, and properties of hyperoctahedral group.

The aforementioned comparisons took place in Paper III. Note, that the formulas regarding complex Wishart matrices $W_{\mathbb{C}}$ and real Wishart matrices $W$ differ for fixed $n$ and $p$. In Table 4.1, $\mathbb{E}\left[\operatorname{Tr}\left\{W_{\bullet}^{k}\right\}\right]$ is presented for $k \leq 5$, where $W_{\bullet}$ denotes either $W_{\mathbb{C}} \sim \mathcal{W}_{p}^{\mathbb{C}}\left(I_{p}, n\right)$ or $W \sim \mathcal{W}_{p}\left(I_{p}, n\right)$. There is a significant difference between the real and complex cases. In particular, it means that (4.10) cannot be applied when $W_{\mathbb{C}}$ is replaced by $W$.

Table 4.1: Comparison of $\mathbb{E}\left[\operatorname{Tr}\left\{W^{k}\right\}\right]$ and $\mathbb{E}\left[\operatorname{Tr}\left\{W_{\mathbb{C}}^{k}\right\}\right]$, when $k \leq 5, W_{\mathbb{C}} \sim \mathcal{W}_{\mathbb{C}, p}\left(I_{p}, n\right)$ and $W \sim \mathcal{W}_{p}\left(I_{p}, n\right)$. The formulas have been derived using (4.7) and (4.10).

\begin{tabular}{lll}
\hline$k$ & $\mathbb{E}\left[\operatorname{Tr}\left\{W^{k}\right\}\right]$ & $\mathbb{E}\left[\operatorname{Tr}\left\{W_{\mathbb{C}}^{k}\right\}\right]$ \\
\hline 1 & $n p$ & $n p$ \\
2 & $n p(n+p+1)$ & $n p(n+p)$ \\
3 & $n p\left(n^{2}+p^{2}+n p+3 n+3 p+4\right)$ & $n p\left(n^{2}+p^{2}+n p+1\right)$ \\
4 & $n p\left(n^{3}+p^{3}+6 n^{2} p+6 n p^{2}+6 n^{2}+\right.$ & $n p\left(n^{3}+p^{3}+6 n^{2} p+6 n p^{2}+5 n+5 p\right)$ \\
& $\left.6 p^{2}+17 n p+21 n+21 p+20\right)$ & \\
5 & $n p\left(p^{4}+n^{4}+10 n p^{3}+10 n^{3} p+20 n^{2} p^{2}+\right.$ & $n p\left(n^{4}+p^{4}+10 n^{3} p+10 n p^{3}+20 n^{2} p^{2}+\right.$ \\
& $61 n^{2}+61 p^{2}+163 n p+10 n^{3}+10 p^{3}+$ & $\left.15 n^{2}+15 p^{2}+40 n p+8\right)$ \\
& $\left.55 n^{2} p+55 n p^{2}+148 n+148 p+132\right)$ & \\
\hline
\end{tabular}

Using (4.10) we have

$$
\mathbb{E}\left[\operatorname{Tr}\left\{W_{\mathbb{C}}^{2}\right\}\right]=\frac{3(n+p)}{3} \mathbb{E}\left[\operatorname{Tr}\left(W_{\mathbb{C}}\right)\right]=n p(n+p),
$$

which differs from the real case, i.e., $\mathbb{E}\left[\operatorname{Tr}\left\{W^{2}\right\}\right]=n p(n+p+1)$. We can also see the difference through a simple example.

Example 4.3.2. Let $X$ be a $2 \times 2$ real matrix such that $X_{i j} \sim N(0,1)$ and let the matrix $W$ be given as

$$
W=X X^{\prime}=\left(\begin{array}{cc}
X_{11}^{2}+X_{12}^{2} & X_{11} X_{21}+X_{12} X_{22} \\
X_{11} X_{21}+X_{12} X_{22} & X_{22}^{2}+X_{21}^{2}
\end{array}\right) .
$$

Hence, since $W \sim \mathcal{W}_{2}\left(I_{2}, 2\right)$,

$$
\begin{aligned}
\mathbb{E}[\operatorname{Tr} W] & =\mathbb{E}\left[X_{11}^{2}+X_{12}^{2}+X_{22}^{2}+X_{21}^{2}\right]=4, \\
\mathbb{E}\left[\operatorname{Tr}\left\{W^{2}\right\}\right] & =\mathbb{E}\left[\left(X_{11}^{2}+X_{12}^{2}\right)^{2}+2\left(X_{11} X_{21}+X_{12} X_{22}\right)^{2}+\left(X_{22}^{2}+X_{21}^{2}\right)^{2}\right]=20,
\end{aligned}
$$

which is consistent with the formulas (4.4) and (4.5). Similarly, let us consider the case of a $2 \times 2$ complex matrix $X$ such that $X_{j k}=\frac{Y_{j k}+\mathfrak{i} Z_{j k}}{\sqrt{2}}, Y_{j k}, Z_{j k} \sim$ 
$N(0,1)$ and $Y_{j k}, Z_{j k}$ are independent for all $j, k=1,2$. Then, $X_{j k}$ has mean 0 and variance 1 and the matrix $W_{\mathbb{C}}=X X^{*} \sim \mathcal{W}_{2}^{\mathbb{C}}\left(I_{2}, 2\right)$. Furthermore,

$$
\begin{aligned}
& \mathbb{E}\left[\operatorname{Tr} W_{\mathbb{C}}\right]=\frac{1}{2} \mathbb{E}\left[Y_{11}^{2}+Z_{11}^{2}+Y_{12}^{2}+Z_{12}^{2}+Y_{21}^{2}+Z_{21}^{2}+Y_{22}^{2}+Z_{22}^{2}\right]=4, \\
& \mathbb{E}\left[\operatorname{Tr}\left\{W_{\mathbb{C}}^{2}\right\}\right]=\frac{1}{4} \mathbb{E}\left[\left(Y_{11}^{2}+Z_{11}^{2}+Y_{12}^{2}+Z_{12}^{2}\right)^{2}+2\left(Y_{12} Y_{22}+Z_{12} Z_{22}+Y_{11} Y_{21}+Z_{11} Z_{21}\right)^{2}\right. \\
& \left.\quad+2\left(Z_{12} Y_{22}-Y_{12} Z_{22}+Z_{11} Y_{21}-Y_{11} Z_{21}\right)^{2}+\left(Y_{21}^{2}+Z_{21}^{2}+Y_{22}^{2}+Z_{22}^{2}\right)^{2}\right]=16 .
\end{aligned}
$$

The difference between the results for the real and complex Wishart matrices of fixed size increases together with the power $k$. It can be observed that it is given by a polynomial of degree lower than $k+1$.

Moreover, difference in the results, regarding expectation of products of trace, for real and complex Wishart matrices is discussed in Graczyk et al. (2005). In the latter paper, the approach used is based on, e.g., the representation theory and graph theory.

\subsubsection{Asymptotic distribution of $\frac{1}{p} \operatorname{Tr}\left\{\left(\frac{1}{n} X X^{\prime}\right)^{t}\right\}$}

The normalized trace of powers of the Wishart matrix, namely $\frac{1}{p} \operatorname{Tr}\left\{\left(\frac{1}{n} X X^{\prime}\right)^{t}\right\}$, is an interesting object from several perspectives. As mentioned earlier, the expectation of $\frac{1}{p} \operatorname{Tr}\left\{\left(\frac{1}{n} X X^{\prime}\right)^{t}\right\}$ gives the $t$ th moment of the Marčenko-Pastur distribution. Having knowledge about the free moments of $\frac{1}{n} X X^{\prime}$ (free cumulants) and putting $\tau(\cdot)=\frac{1}{p} \mathbb{E}[\operatorname{Tr}\{\cdot\}]$ we obtain knowledge about the spectral distribution of that matrix. Furthermore, we show, using the formula (4.8), that it converges to its expectation for any $t$. The steps below lead us to the conclusion that all cumulants, beside the mean, vanish asymptotically under Kolmogorov condition. Thus, it is shown that $\frac{1}{p} \operatorname{Tr}\left\{\left(\frac{1}{n} X X^{\prime}\right)^{t}\right\}$ converges in probability to its mean, i.e., is a consistent estimator. Presented in this section a proof was derived by the author although it is not a part of any of the appended papers.

Lemma 4.3.1. Assume that $X X^{\prime} \sim \mathcal{W}_{p}\left(I_{p}, n\right)$ and that the Kolmogorov condition holds, i.e., $\lim _{p, n \rightarrow \infty} \frac{n}{p}=c$. Then,

$$
\lim _{n, p \rightarrow \infty} \mathbb{E}\left[\left(\frac{1}{p} \operatorname{Tr}\left\{\left(\frac{1}{n} X X^{\prime}\right)^{t}\right\}\right)^{k}\right]=p_{t}(c)^{k}, \quad t \in \mathbb{N},
$$

where $p_{t}(c):=\lim _{n, p \rightarrow \infty} \frac{1}{p} \mathbb{E}\left[\operatorname{Tr}\left\{\left(\frac{1}{n} X X^{\prime}\right)^{t}\right\}\right]$, i.e., is a polynomial in $c$ given by the limit of the first moment.

Proof. Put

$$
m_{k}^{(t)}=\lim _{n, p \rightarrow \infty} E\left[\left(\frac{1}{p} \operatorname{Tr}\left\{\left(\frac{1}{n} X X^{\prime}\right)^{t}\right\}\right)^{k}\right]
$$


Thus,

$$
m_{k}^{(t)}=\lim _{n, p \rightarrow \infty} \frac{1}{p^{k} n^{t k}} E\left[\left(\operatorname{Tr}\left\{\left(X X^{\prime}\right)^{t}\right\}\right)^{k}\right]=Q^{\{0, \overbrace{t, \ldots, t}^{k-1}}(t)
$$

where used notation $Q^{\{0, \overbrace{t, \ldots, t}^{k-1}\}}(t)$ is already defined in Subsection 4.3.1. Let us firstly consider the case when $t=1$. Then,

$$
\begin{aligned}
& m_{1}^{(1)}=Q^{\{0\}}(1)=1, \\
& m_{k}^{(1)}=Q^{\{0, \overbrace{1, \ldots, 1}^{k-1}\}}(1)=Q^{\{0, \overbrace{1, \ldots, 1}^{k-2}\}}(1)=m_{k-1}^{(1)}, \\
& \frac{m_{k}^{(1)}}{m_{k-1}^{(1)}}=1 .
\end{aligned}
$$

Hence, $p_{1}(c)=1$ and $m_{k}^{(1)}=1$ for all $k \in \mathbb{N}$. Now put $t=2$. In this case

$$
\begin{aligned}
m_{1}^{(2)} & =Q^{\{0\}}(2)=\left(1+\frac{1}{c}\right) Q^{\{0\}}(1)=1+\frac{1}{c}, \\
m_{k}^{(2)} & =Q^{\{0, \overbrace{2, \ldots, 2}^{k-1}\}}(2)=\left(1+\frac{1}{c}\right) Q^{\{0, \overbrace{2, \ldots, 2}^{k-1}}(1)=\left(1+\frac{1}{c}\right) Q^{\{0, \overbrace{2, \ldots, 2}^{k-2}} \\
& =\left(1+\frac{1}{c}\right) m_{k-1}^{(2)}, \\
\frac{m_{k}^{(2)}}{m_{k-1}^{(2)}} & =1+\frac{1}{c} .
\end{aligned}
$$

Hence, $p_{2}(c)=1+\frac{1}{c}$ and $m_{k}^{(2)}=\left(1+\frac{1}{c}\right)^{k}$ for all $k \in \mathbb{N}$. To prove the statement for any $t$, let it be chosen arbitrary. Thus, if we put $p_{t}(c):=m_{1}^{(t)}=Q^{\{0\}}(t)$ and use the recursive formula with respect to $t$ given in (4.8) it leads to the conclusion that $Q^{\{0, \overbrace{t, \ldots, t}^{k-1}\}}(t)$ is reduced to $Q^{\{0, \overbrace{t, \ldots, t}^{k-2}\}}(t)$ with exactly the same recursive steps with respect to $t$ as when calculating $p_{t}(c)$. As the limit of the first moment exists, we use the algebraic limit theorem $\lim _{x \rightarrow \infty}(f(x) \cdot g(x))=\lim _{x \rightarrow \infty} f(x)$. $\lim _{x \rightarrow \infty} g(x)$ to establish that

$$
\left.m_{k}^{(t)}=Q^{\{0, \overbrace{t, \ldots, t}^{k-1}\}}(t)=p_{t}(c) Q^{\{0, \overbrace{t, \ldots, t}^{k-2}}\right\}(t)=p_{t}(c) m_{k-1}^{(t)} .
$$

Consequently,

$$
\frac{m_{k}^{(t)}}{m_{k-1}^{(t)}}=p_{t}(c)
$$


which leads to $m_{k}^{(t)}=p_{t}(c)^{k}$ for any $t, k \in \mathbb{N}$, i.e., to the statement of Lemma 4.3.1.

Lemma 4.3.2. Assume that $X X^{\prime} \sim \mathcal{W}_{p}\left(I_{p}, n\right)$ and that the Kolmogorov condition holds, i.e., $\lim _{p, n \rightarrow \infty} \frac{n}{p}=c$. Then, $c_{i}^{(t)}$ denoting an asymptotic value of the ith cumulant of $\frac{1}{p} \operatorname{Tr}\left\{\left(\frac{1}{n} X X^{\prime}\right)^{t}\right\}$ is equal to zero for $i=2,3, \ldots$.

Proof. Let us first indicate that $c_{k}$ and $m_{k}$ stand for the asymptotic value of cumulants of degree $k$ and the asymptotic value of the moments of degree $k$, respectively.

One of the well-know moment-cumulant relation formulas, see DasGupta (2008), states that

$$
c_{k}^{(t)}=m_{k}^{(t)}-\sum_{p=1}^{k-1}\left(\begin{array}{l}
k-1 \\
k-p
\end{array}\right) c_{p}^{(t)} m_{k-p}^{(t)} .
$$

By Lemma 4.3.1, the $k$ th moment is given by the $k$ th power of the polynomial in $c$, i.e., $m_{k}^{(t)}=p_{t}(c)^{k}$ for any $t, k \in \mathbb{N}$. Therefore,

$$
\begin{aligned}
& c_{1}^{(t)}=m_{1}^{(t)}=p_{t}(c), \\
& c_{2}^{(t)}=m_{2}^{(t)}-c_{1}^{(t)} m_{1}^{(t)}=p_{t}(c)^{2}-\left(p_{t}(c)\right)^{2}=0, \\
& c_{3}^{(t)}=m_{3}^{(t)}-\left(\begin{array}{l}
2 \\
2
\end{array}\right) c_{1}^{(t)} m_{2}^{(t)}-\left(\begin{array}{l}
2 \\
1
\end{array}\right) c_{2}^{(t)} m_{1}^{(t)}=p_{t}(c)^{3}-\left(p_{t}(c)\right)^{3}-0=0 .
\end{aligned}
$$

We put an induction assumption that all cumulants of a degree of at least 2 and at most $k$ are equal to zero, i.e., $c_{i}^{(t)}=0$ for $i=2, \ldots, k$. Then

$$
\begin{aligned}
c_{k+1}^{(t)} & =m_{k+1}^{(t)}-\sum_{p=1}^{k}\left(\begin{array}{c}
k \\
k+1-p
\end{array}\right) c_{p}^{(t)} m_{k+1-p}^{(t)} \\
& =p_{t}(c)^{k+1}-\left(\begin{array}{l}
k \\
k
\end{array}\right) c_{1}^{(t)} m_{k}^{(t)}-\sum_{p=2}^{k}\left(\begin{array}{c}
k \\
k+1-p
\end{array}\right) \underbrace{c_{p}^{(t)}}_{=0} m_{k+1-p}^{(t)} \\
& =p_{t}(c)^{k+1}-p_{t}(c) p_{t}(c)^{k}=0 .
\end{aligned}
$$

Hence, the statement is true for all cumulants of a degree of at least 2, i.e., $c_{i}^{(t)}=0$ for all $i=2,3, \ldots$

By Lemma 4.3.2 the only non-zero cumulant of $\frac{1}{p} \operatorname{Tr}\left\{\left(\frac{1}{n} X X^{\prime}\right)^{t}\right\}$ is $c_{1}^{(t)}=p_{t}(c)$, for $t \in \mathbb{N}$. Hence, we have proved that $\frac{1}{p} \operatorname{Tr}\left\{\left(\frac{1}{n} X X^{\prime}\right)^{t}\right\}$ converges in probability to its mean, as claimed in the beginning of the section.

Since the studied object $\frac{1}{p} \operatorname{Tr}\left\{\left(\frac{1}{n} X X^{\prime}\right)^{t}\right\}$ has variance 0 , we suggest the idea of scaling that will result in an asymptotically normally distributed object. 
Let $W \sim \mathcal{W}_{p}\left(I_{p}, n\right)$, then the distribution of $\left(\eta_{1}, \ldots, \eta_{m}\right)$, where

$$
\eta_{k}=\frac{1}{(n+p)^{k}}\left(\operatorname{Tr}\left\{W^{k}\right\}-\mathbb{E}\left[\operatorname{Tr}\left\{W^{k}\right\}\right]\right)
$$

has been proved to converge to the multivariate normal distribution by Jonsson (1982). He provides a combinatorial proof based on the method of moments as suggested in Arharov (1971). In the latter paper, proofs are not given. Jonsson also improved scaling of the random variable, in relation to Arharov's paper, where $(n+p)^{k / 2} \eta_{k}$ was considered. Although the statement of Theorem 4.1 in the paper by Jonsson (1982) regarding multivariate normal distribution is correct, the proof is quite difficult to follow. Moreover, the variance is not given explicitly and later papers applying Jonsson's results, e.g., see Ledoit and Wolf (2002), must use the results for moments given in, for example, John (1972).

In Paper IV a scaled version of the normalized trace of powers of Wishart matrices, which is of the form

$$
Y_{k}=\sqrt{n p}\left(\frac{1}{p} \operatorname{Tr}\left\{\left(\frac{1}{n} W\right)^{k}\right\}-m_{1}^{(k)}(n, p)\right),
$$

where $m_{1}^{(k)}(n, p)=\mathbb{E}\left[\frac{1}{p} \operatorname{Tr}\left\{\left(\frac{1}{n} W\right)^{k}\right\}\right]$, has been considered in the case of $k=1$ and $k=2$. It is indicated how to prove that the vector $Y=\left(Y_{1}, Y_{2}\right)$ follows an asymptotic multivariate normal distribution. The result can be extended to higher dimensions. The random variables $\eta_{k}$ and $Y_{k}$ differ by a constant $\sqrt{c} /(1+c)$ in $p / n$-asymptotics. In this approach, one is using the cumulants and the moments of a random variables $Y_{i}$ considered as a polynomial with respect to $\frac{1}{p}$. Then, the coefficients of polynomials are determined in finite and asymptotic regime. The results are under the scaling $\sqrt{n p}$ that is a symmetric function, with respect to $p$ and $n$ (compare the scaling by Ledoit and Wolf, 2002) and give the covariance matrix that makes results suitable to be used in future applications, for example, in hypothesis testing. For more details regarding the proof, see Paper IV.

\subsection{Testing the identity of covariance matrices}

Considering possible applications of results regarding multivariate normality of the previous section based on Paper IV as well as on the results by Jonsson (1982), we came to investigate hypothesis testing problems connected to Wishart matrices. As we have seen in Subsection 4.2, the sample covariance matrix under normality of the matrix $X$ follows a Wishart distribution.

In this section as in Paper IV, we are interested in an identity test of the covariance matrix that works in the case where the dimension $p$ exceeds, is equal or is smaller than the sample size $n$, i.e., $p>n, p=n$ or $p<n$. We 
assume normality of data represented in a $p \times n$ matrix $X$ and that the Kolmogorov condition holds, so that both $p$ and $n$ increase with the same speed, i.e., $\lim _{n, p \rightarrow \infty} \frac{n}{p}=c \in(0, \infty)$. The hypothesis for testing identity is given by

$$
H_{0}: \Sigma=I_{p} \text { against } H_{1}: \Sigma \neq I_{p}
$$

which of course is the same as

$$
H_{0}: \Sigma=\Sigma_{0} \text { against } H_{1}: \Sigma \neq \Sigma_{0},
$$

where the matrix $\Sigma_{0}$ is a given positive definite matrix. Equivalence of both formulations holds, since we can consider the transformation $\Sigma_{0}^{-\frac{1}{2}} X$ instead of the data matrix $X$.

Mauchly (1940) tested the hypothesis (4.11) using a likelihood ratio approach. Tests based on likelihood ratio statistics were long commonplace, but they are only suitable in the case where $p<n$, see Muirhead (1982) and Anderson (2003). There is a number of test statistics based on the estimators of $a_{1}=\frac{1}{p} \sum_{i=1}^{p} \lambda_{i}$ and $a_{2}=\frac{1}{p} \sum_{i=1}^{p} \lambda_{i}^{2}$, where $\lambda_{i}$ are eigenvalues of $\frac{1}{n} X X^{\prime}$. One of the early ones is a result given by Nagao (1973). He, instead for Kolmogorov condition with both $n, p \rightarrow \infty$, assumed that $p$ remains constant while $n$ increases. Further investigation, of given by him test statistic, showed that the limiting distribution differs from the original results when assuming the Kolmogorov condition to be true. In the paper by Ledoit and Wolf (2002) a modification of Nagao's test statistics is given as

$$
T_{W}=\frac{1}{p} \operatorname{Tr}\left\{\left(\frac{1}{n} X X^{\prime}-I_{p}\right)^{2}\right\}-\frac{p}{n}\left(\frac{1}{p} \operatorname{Tr}\left\{\frac{1}{n} X X^{\prime}\right\}\right)^{2}+\frac{p}{n} .
$$

This test statistic is consistent, unlike the Nagao's result, for $p>n$. Indeed, both Nagao's and $T_{W}$ statistics have the same behavior when $p$ is fixed, but $T_{W}$, in contradiction to the earlier version, is robust under high-dimensionality. Furthermore, the result of Ledoit and Wolf (2002) has been further developed for $\lim _{n, p \rightarrow \infty} \frac{n}{p}=c>0$ by Fujikoshi et al. (2011) and Birke and Dette (2005). There exists approaches, that origins in Nagao's test statistics, when the normality assumption is omitted, see paper by Chen et al. (2010). The publication by Srivastava (2005) also follows the paper of Nagao (1973). Srivastava (2005) proposes a test statistic of the form

$$
T_{S}=\frac{n}{2}\left(\hat{a}_{2}-2 \hat{a}_{1}+1\right),
$$

where

$$
\begin{aligned}
& \hat{a}_{1}=\frac{1}{p} \operatorname{Tr}\left\{\frac{1}{n} X X^{\prime}\right\} \\
& \hat{a}_{2}=\frac{n^{2}}{(n-1)(n+2) p}\left[\operatorname{Tr}\left\{\frac{1}{n^{2}}\left(X X^{\prime}\right)^{2}\right\}-\frac{1}{n}\left(\operatorname{Tr}\left\{\frac{1}{n} X X^{\prime}\right\}\right)^{2}\right]
\end{aligned}
$$


are asymptotic normally distributed, unbiased and consistent, under the Kolmogorov condition, estimators of $a_{1}$ and $a_{2}$. Note that Srivastava assumed that $n=O\left(p^{\delta}\right)$, where $0<\delta \leq 1$, hence his results include both the case $\lim _{n, p \rightarrow \infty} \frac{n}{p}=0$ and $\lim _{n, p \rightarrow \infty} \frac{n}{p}=c \in(0, \infty)$. Furthermore, under the same conditions, the spherecity test for the covarinace matrix and tests for the hypothesis that the covarinace matrix is a diagonal matrix are provided by Srivastava (2005).

The test statistics given by Fisher et al. (2010) and Fisher (2012) are a natural continuation of Srivastava's research. They propose the following test statistics

$$
\begin{aligned}
& T_{1}=\frac{n}{c \sqrt{8}}\left(\hat{a}_{4}-4 \hat{a}_{3}+6 \hat{a}_{2}-4 \hat{a}_{1}+1\right), \\
& T_{2}=\frac{n}{\sqrt{8\left(c^{2}+12 c+8\right)}}\left(\hat{a}_{4}-2 \hat{a}_{2}+1\right)
\end{aligned}
$$

that are asymptotic normally distributed, and based on the unbiased and consistent, under the Kolmogorov condition, estimators of $a_{j}=\frac{1}{p} \sum_{i=1}^{p} \lambda_{i}^{j}$, for $i=1,2,3,4$.

Srivastava (2005), Fisher et al. (2010) and Fisher (2012) based their test statistics on the idea that the null hypothesis $\Sigma=I_{p}$, implies that all eigenvalues equal 1. Then

$$
\frac{1}{p} \sum_{i=1}^{p}\left(\lambda_{i}-1\right)^{2 k}=\frac{1}{p} \sum_{i=1}^{p} \sum_{j=0}^{2 k}(-1)^{j}\left(\begin{array}{c}
2 k \\
j
\end{array}\right) \lambda_{i}^{2 k-j}=\sum_{j=0}^{2 k}(-1)^{j}\left(\begin{array}{c}
2 k \\
j
\end{array}\right) a_{2 k-j}
$$

and

$$
\frac{1}{p} \sum_{i=1}^{p}\left(\lambda_{i}^{2}-1\right)^{2 k}=\frac{1}{p} \sum_{i=1}^{p} \sum_{j=0}^{2 k}(-1)^{j}\left(\begin{array}{c}
2 k \\
j
\end{array}\right) \lambda_{i}^{2(2 k-j)}=\sum_{j=0}^{2 k}(-1)^{j}\left(\begin{array}{c}
2 k \\
j
\end{array}\right) a_{4 k-2 j}
$$

are non-negative as they are the sum of even powers of $\lambda_{i}-1$ and $\lambda_{i}^{2}-1$, respectively. We can also easily see that under $H_{0}$

$$
\frac{1}{p} \sum_{i=1}^{p}\left(\lambda_{i}-1\right)^{2 k}=0 \quad \text { and } \quad \frac{1}{p} \sum_{i=1}^{p}\left(\lambda_{i}^{2}-1\right)^{2 k}=0 .
$$

In Paper IV, using the normality of $Y_{t}=\sqrt{n p}\left(\frac{1}{p} \operatorname{Tr}\left\{\left(\frac{1}{n} X X^{\prime}\right)^{t}\right\}-m_{1}^{(t)}(n, p)\right)$ for all $t=1, \ldots, m$, we test the hypothesis (4.11) through a goodness-of-fit approach with the test statistic

$$
T_{J m}=Y^{\prime} \Sigma_{Y}^{-1} Y \sim \chi^{2}(m),
$$

where $Y=\left(Y_{1}, \ldots, Y_{m}\right)$ for $m=2,3,4$. Here, $\Sigma_{Y}$ denotes the asymptotic covariance matrix of the vector $Y$ given by $\Sigma_{Y}=\left(\operatorname{Cov}\left(Y_{i}, Y_{j}\right)\right)_{i, j=1}^{m}$ with elements of the matrix calculated through (4.7), which is a result of Paper III. It has very good power compared to the tests of Srivastava, Fisher and Ledoit and Wolf with a similar attained significant level. Let us see some of the data simulated for the performed comparison. In Table 4.2 we analyze the attained significance levels, while Table 4.3 gives empirical statistical power. 
Table 4.2: Comparison of empirical significance levels. $T_{J 2}, T_{J 3}, T_{J 4}$ denote test statistics introduced by the author, see (4.12), $T_{W}$ stands for the Ledoit and Wolf test statistic, $T_{S}$ for Srivastava's test statistic and $T_{1}$ and $T_{2}$ for the two test statistics introduced by Fisher.

\begin{tabular}{|c|c||c|c|c||c|c|c|c|}
\hline \multirow{2}{*}{} & \multicolumn{1}{|c||}{$\alpha$} & \multicolumn{3}{c||}{$T_{J}$} & \multicolumn{4}{c|}{ Alternative tests } \\
\cline { 2 - 9 } & $\alpha$ & $T_{J 2}$ & $T_{J 3}$ & $T_{J 4}$ & $T_{W}$ & $T_{S}$ & $T_{1}$ & $T_{2}$ \\
\hline \multirow{2}{*}{$\begin{array}{l}p=125 \\
n=250\end{array}$} & .1 & .097 & .093 & .0935 & .1045 & .104 & .097 & .0995 \\
\cline { 2 - 9 } & .05 & .0405 & .051 & .0515 & .049 & .048 & .058 & .0565 \\
\cline { 2 - 9 } & .025 & .021 & .0225 & .028 & .024 & .023 & .0305 & .0325 \\
\cline { 2 - 9 } & .01 & .009 & .0075 & .0135 & .0095 & .0095 & .0155 & .0175 \\
\hline \hline \multirow{3}{*}{$\begin{array}{l}p=250 \\
n=125\end{array}$} & .1 & .1065 & .098 & .0955 & .107 & .106 & .107 & .103 \\
\cline { 2 - 9 } & .05 & .0505 & .0455 & .0495 & .053 & .0515 & .058 & .056 \\
\cline { 2 - 9 } & .025 & .024 & .023 & .028 & .0285 & .028 & .0305 & .0315 \\
\cline { 2 - 9 } & .01 & .0085 & .01 & .0135 & .013 & .012 & .012 & .0135 \\
\hline
\end{tabular}

Power studies are performed for the alternative hypothesis that data matrix is an observation from a random matrix $X \sim \mathcal{N}_{p, n}\left(0, \Sigma, I_{n}\right)$, where covariance ma-

Table 4.3: Comparison of empirical powers of tests for $\alpha=0.05 . T_{J 2}, T_{J 3}, T_{J 4}$ denote test statistics introduced by the author, see (4.12), $T_{W}$ stands for the Ledoit and Wolf test statistic, $T_{S}$ for Srivastava test statistic, $T_{1}$ and $T_{2}$ for the two test statistics introduced by Fisher.

\begin{tabular}{|c|c|c|c|c|c|c|c|c|}
\hline & \multicolumn{3}{|c|}{$T_{J}$} & \multicolumn{4}{|c|}{ Alternative tests } \\
\hline & & $T_{J 2}$ & $T_{J 3}$ & $T_{J 4}$ & $T_{W}$ & $T_{S}$ & $T_{1}$ & $T_{2}$ \\
\hline \multirow{3}{*}{$\begin{array}{l}p=125 \\
n=125\end{array}$} & $\Sigma=1.005^{2} I$ & .1195 & .121 & .13 & .059 & .0585 & .0685 & .081 \\
\hline & $\Sigma=1.01^{2} I$ & .2855 & .296 & .2855 & .054 & .0525 & .0705 & .072 \\
\hline & $\Sigma=1.03^{2} I$ & .9985 & .9975 & .9935 & .124 & .1135 & .102 & .130 \\
\hline \multirow{3}{*}{$\begin{array}{l}p=125 \\
n=250\end{array}$} & $\Sigma=1.005^{2} I$ & .19 & .16 & .152 & .048 & .0465 & .0595 & .061 \\
\hline & $\Sigma=1.01^{2} I$ & .5995 & .534 & .504 & .071 & .069 & .07 & .080 \\
\hline & $\Sigma=1.03^{2} I$ & 1 & 1 & 1 & .175 & .159 & .111 & .175 \\
\hline \multirow{3}{*}{$\begin{array}{l}p=250 \\
n=125\end{array}$} & $\Sigma=1.005^{2} I$ & .194 & .171 & .163 & .052 & .049 & .0655 & .070 \\
\hline & $\Sigma=1.01^{2} I$ & .6155 & .551 & .512 & .068 & .064 & .069 & .067 \\
\hline & $\Sigma=1.03^{2} I$ & 1 & 1 & 1 & .117 & .1045 & .102 & .115 \\
\hline \multirow[t]{3}{*}{$\begin{array}{l}p=125 \\
n=125\end{array}$} & $\begin{array}{c}\Sigma=\operatorname{diag}(U), \\
U \sim U(1 \pm .01)^{2}\end{array}$ & .1405 & .127 & .118 & .059 & .058 & .0575 & .063 \\
\hline & $\begin{array}{c}\Sigma=\operatorname{diag}(U) \\
U \sim U(1 \pm .1)^{2}\end{array}$ & .885 & .875 & .87 & .259 & .265 & .0815 & .142 \\
\hline & $\begin{array}{c}\Sigma=\operatorname{diag}(U) \\
U \sim U(1 \pm .3)^{2}\end{array}$ & .961 & .9575 & .956 & .748 & .747 & .2715 & .659 \\
\hline
\end{tabular}


trix if given by:

- $\Sigma=a I_{p}$ for fixed $a$ close to 1 , see Table 4.3 ;

- $\Sigma=a I_{p}$ for $a \sim U(1-\varepsilon, 1+\varepsilon)$, see Table 4.3 ;

- $\Sigma=\left(\begin{array}{cc}a I_{k} & 0 \\ 0 & I_{p-k}\end{array}\right)$ as well as $\Sigma=\left(\begin{array}{cc}I_{p / 2} & a J_{p / 2} \\ a J_{p / 2} & I_{p / 2}\end{array}\right)$, where $J_{p / 2}$ stands for a matrix of ones of size $\frac{p}{2} \times \frac{p}{2}$, see Paper IV.

Indeed, in Table 4.2 and 4.3, we see that the test proposed in Paper IV provides much better empirical power than the alternative methods and keeps a similar performance with respect to the size of the test. With $\Sigma=1.03^{2} I_{p}$, we are able to reject $H_{0}: \Sigma=I_{p}$ with probability 1 , while the other tests reach a power of maximum of $17.5 \%$. The test performance remains good when the elements of the diagonal covariance matrix come from a uniform distribution in an interval surrounding 1 .

Furthermore, other methods to test the hypothesis (4.11) allowing for large dimension $p$ are given in, among others, Schott (2001, 2005, 2007), Bai et al. (2009), Jiang et al. (2012), Jiang and Yang (2013) and Jiang and Qi (2015). 



\section{Summary of papers, conclusions and further research}

The purpose of this chapter is to give an overview of the results in the papers contributed to the thesis and discuss future work.

\section{$5.1 \quad$ Summary of papers}

\section{Paper I: Closed form of the asymptotic spectral distribution of random matrices using free independence}

The spectral distribution function of random matrices is an information-carrying object widely studied within Random matrix theory. Random matrix theory is the main field placing its focus on the diverse asymptotic properties of the matrices, with a particular emphasis placed on the eigenvalue distribution. The aim of this article is to point out some classes of matrices, which have closed form expressions for the asymptotic spectral distribution function. We consider matrices, later denoted by $\mathcal{Q}$, which can be decomposed into the sum of asymptotically free independent summands.

Let $(\Omega, \mathcal{F}, P)$ be a probability space. We consider the particular example of a non-commutative space $\left(R M_{p}(\mathbb{C}), \tau\right)$, where $R M_{p}(\mathbb{C})$ denotes the set of all $p \times p$ random matrices, with entries which are complex random variables with finite moments of any order and $\tau$ is a tracial functional. In particular, explicit calculations are performed in order to generalize the theorem given by Marčenko and Pastur (1967) and illustrate the use of asymptotic free independence to obtain the asymptotic spectral distribution for a particular form of the matrix $Q \in \mathcal{Q}$.

Finally, the main result is a new theorem pointing out classes of the matrix $Q$, which leads to a closed formula for the asymptotic spectral distribution. Formulation of results for matrices with inverse Stieltjes transforms, with respect to the composition, given by a ratio of a 1 st and 2 nd degree polynomials, is provided. 


\section{Paper II: Cumulant-moment relation in free probability theory}

The goal of this paper is to present and prove a new cumulant-moment recurrent relation formula within Free probability theory. It is a convenient tool to determine an underlying compactly supported distribution function. The existing recurrent relations between these objects require the combinatorial understanding of the idea of non-crossing partitions, which has been considered by Nica and Speicher (2006). Furthermore, some formulations are given with additional use of the Möbius function. The recursive result derived in this paper does not require introducing any of those concepts. Similarly to the non-recursive formulation of Mottelson (2012), our formula only demands summing over integer non-negative partitions of the number, i.e., for integer $t$ over all non-negative integers $a_{1}, \ldots, a_{k}$ such that $\sum_{i=1}^{k} a_{i}=t$ for all $k=1, \ldots, t$. The proof of a non-recurrent result is given using the Lagrange inversion formula, while in our proof the calculations of the Stieltjes transform of the underlying measure are essential.

\section{Paper III: On $\mathbb{E}\left[\prod_{i=0}^{k} \operatorname{Tr}\left\{W^{m_{i}}\right\}\right]$, where $W \sim \mathcal{W}_{p}(I, n)$}

In this paper, we give a general recursive formula for $\mathbb{E}\left[\prod_{i=0}^{k} \operatorname{Tr}\left\{W^{m_{i}}\right\}\right]$, where $W \sim \mathcal{W}_{p}(I, n)$ denotes a real Wishart matrix. Formulas for fixed $n, p$ are presented, as well as asymptotic versions where $\frac{n}{p} \stackrel{n, p \rightarrow \infty}{\rightarrow} c$, i.e., when the so-called Kolmogorov condition holds. Finally, we show the application of the asymptotic moment relation when deriving moments for the Marčenko-Pastur distribution (free Poisson law). A numerical illustration using the implementation of the main result is also performed.

\section{Paper IV: On $p / n$-asymptotics applied to traces of 1st and 2nd order powers of Wishart matrices with application to goodness-of-fit testing}

The distribution of the vector of the normalized traces of $\frac{1}{n} X X^{\prime}$ and of $\left(\frac{1}{n} X X^{\prime}\right)^{2}$, where the matrix $X: p \times n$ follows a matrix normal distribution and it is proved, under the Kolmogorov condition $\frac{n}{p} \stackrel{n, p \rightarrow \infty}{\rightarrow} c>0$, to be multivariate normally distributed. Asymptotic moments and cumulants are obtained using a recursive formula derived in Paper III. We use this result to test for identity of the covariance matrix using a goodness-of-fit approach. The test performs well regarding the power compared to presented alternatives, for both $c<1$ or $c \geq 1$. 


\subsection{Conclusions}

The assumption made in the research papers presented in the previous section is that the Kolmogorov condition holds, i.e., $\lim _{n, p \rightarrow \infty} \frac{p}{n}=c \in(0, \infty)$. From a practical point of view, the asymptotic approach allows us to build results which are suitable for the analysis of high-dimensional data sets (or multivariate data sets in the case of $c \leq 1$ ). From the other perspective, this convenient set-up allows us to use properties such as asymptotic freeness and its implications, see Paper I. We have tried to show the strong connection between random matrices and free probability theory that was established with famous papers by Voiculescu $(1985,1991)$. Tools such as the Stieltjes and R-transforms are presented in application to the random matrices-related research questions, such as deriving eigenvalue distribution, as well as their connection to the bigger picture of free probability.

Furthermore, using the relation between free moments, free cumulants and the Stieltjes and $\mathrm{R}$-transforms, we have proved an alternative free momentcumulant formula that do not require summation over the non-crossing partitions of the set. Since, the R-transform can be used to define the free cumulants, the concept of the non-crossing partitions could be omitted. Although, of course, it brings beautiful connection to, e.g., the combinatorics and classical probability theory when it corresponding formula we sum over all partitions of the set. Nevertheless, if one decides to approach the free moments and cumulant without using the non-crossing partitions, the new free moment-cumulant formula completes the free probability theory. In Paper II, we give detailed computations on the free cumulants to visualize the advantage of the introduced relation.

In Paper III the expectation of $\prod_{i=0}^{k} \operatorname{Tr}\left\{W^{m_{i}}\right\}$ is studied. The formula for finite $n$ and $p$ is given, as well as the asymptotic results. Our formula is proved for the real Wishart matrices with use of the differentiation with respect to the inverse of a covariance matrix. The result differs from the one obtained for complex Wishart matrices, although for properly scaled Wishart matrices the difference will converge to zero as $n, p \rightarrow \infty$. The formula can be used to re-derive moments of the Marčenko-Pastur law, and hence the recursive relation between Catalan numbers. In the theoretical introduction to papers, see Subsection 4.3.4, we show that using the asymptotic formula given in Paper III we can prove the normality of the $\prod_{i=0}^{k} \operatorname{Tr}\left\{W^{m_{i}}\right\}$. Moreover, one can see that $\mathbb{E}\left[\prod_{i=0}^{k} \operatorname{Tr}\left\{W^{m_{i}}\right\}\right]$ is a special case of the generalized moments described by Capitaine and Casalis (2004). In the latter paper the convergence of generalized moments was used to prove asymptotic freeness, for example, between two complex Wishart matrices. The convergence of moments of the spectral measure was also used to prove the results of Arharov (1971) and Jonsson (1982).

There are a number of papers that refer to the results given by Jonsson (1982). He used the methods proposed earlier by Arharov (1971) but applied them to differently scaled objects. The approach in both publications was to investigate 
the convergence of the mixed moments to the mixed moments of the multivariate normal distribution. In Paper IV we present an alternative proof of multivariate normality for $\left(Y_{1}, Y_{2}\right)=\left(\frac{1}{p} \operatorname{Tr}\left\{\frac{1}{n} X X^{\prime}\right\}, \frac{1}{p} \operatorname{Tr}\left\{\left(\frac{1}{n} X X^{\prime}\right)^{2}\right\}\right)$ that is based on the knowledge of asymptotic cumulants. We identify, in an explicit manner, the coefficients that cause the vanishing of the asymptotic moments of odd orders, and the value obtained for the even ones. We also carefully investigate the asymptotic cumulants, and through the result of Marcinkiewicz (1939) we show marginal normality of the vector. Multivariate normality is the result of further investigation of arbitrary linear combination of $Y_{1}$ and $Y_{2}$. Moreover, we want to point out that the presented proofs of marginal and multivariate normality are expendable to the vector $\left(Y_{1}, \ldots, Y_{m}\right)$ of arbitrary length, where $Y_{t}=\sqrt{n p}\left(\frac{1}{p} \operatorname{Tr}\left\{\left(\frac{1}{n} X X^{\prime}\right)^{t}\right\}-m_{1}^{(t)}(n, p)\right)$ for all $t=1, \ldots, m$.

The hypothesis testing presented in the last paper is an application of the results obtained on the distribution of $Y_{t}$. Moreover, our approach to testing $H_{0}: \Sigma=I$ is different compared to e.g., Srivastava (2005) and Fisher et al. (2010). They based their derivations on the normally distributed test statistics that are a linear combination of the estimates of the spectral moments. The used linear function is obtained by the fact that under $H_{0}$ all the eigenvalues equal 1 . We work with the sum of squares of differences between the expected and obtained spectral moments, which implies an approximative $\chi^{2}$-distribution of the test statistics.

\subsection{Further research}

Regarding the future work, we plan to complete Paper IV with a proof for arbitrary dimension of vector $Y$. An added value to the paper would be an extension of the simulation study with plots of the power of test for $\Sigma=\kappa I_{p}$ as the function of $\kappa$. That could be used for comparison between proposed test statistics and alternatives.

Moreover, we want to investigate the generalized moments introduced by Capitaine and Casalis (2004) and relate them to the objects studied in Papers III and IV.

We are interested in generalizing the results from the third paper into the formula for Wishart matrices with an arbitrary parameter $\Sigma$.

The aim is also to derive a new sphericity test of the covariance matrix that will behave well in both multivariate and high-dimensional settings. It is my belief, that this test would be of bigger interest than the test derived in the Paper IV.

The ultimate goal is to derive explicit results for the empirical spectral density of sum of random matrices when $\Sigma$ is arbitrary. Girko and von Rosen (1994) presented some equations but they cannot be solved explicitly. 
Moreover, the considered research questions can be evaluated under a variety of the assumptions on the structure of the covariance matrix $\Sigma=\left(\sigma_{i j}\right)_{i, j=1}^{p}$, e.g., Toeplitz $\left(\sigma_{i j}=\sigma_{|i-j|+1}^{2}\right), \operatorname{AR}(1)$ (autoregressive(1), $\sigma_{i j}=\sigma^{2} \rho^{|i-j|}$, where $\rho>0)$, compound symmetry $\left(\sigma_{i j}=\sigma_{1}^{2}+\sigma^{2} \mathbb{1}_{\{i=j\}}\right)$ or variance components ( $\sigma_{i j}=\sigma_{t}^{2}$, where $i$ corresponds to the $t$ th effect) structures. 



\section{References}

Anderson, T. W. (2003). An Introduction to Multivariate Statistical Analysis. Third edition. Wiley Series in Probability and Statistics. Wiley-Interscience, Hoboken, NJ.

Arharov, L. V. (1971). Limits theorems for the characteristical roots of a sample covariance matrix. Soviet Mathematics Doklady, 12:1206-1209.

Bai, Z., Jiang, D., Yao, J., and Zheng, S. (2009). Corrections to LRT on largedimensional covariance matrix by RMT. The Annals of Statistics, 37:38223840 .

Birke, M. and Dette, H. A. (2005). A note on testing the covariance matrix for large dimension. Statistics \&6 Probability Letters, 74:281-289.

Bożejko, M. and Demni, N. (2009). Generating functions of Cauchy-Stieltjes type for orthogonal polynomials. Infinite Dimensional Analysis, Quantum Probability $\&$ Related Topics, 12(1):91-98.

Bryc, W. (2008a). Asymptotic normality for traces of polynomials in independent complex wishart matrices. Probability Theory and Related Fields, 140(3-4):383-405.

Bryc, W. (2008b). Compound real Wishart and q-Wishart matrices. International Mathematics Research Notices, 2008(1):1-42.

Cameron, P. (1998). Introduction to Algebra. Oxford science publications. Oxford University Press.

Capitaine, M. and Casalis, M. (2004). Asymptotic freeness by generalized moments for Gaussian and Wishart matrices. Application to Beta random matrices. Indian University Mathematics Journal, 53:397-432.

Capitaine, M. and Casalis, M. (2006). Cumulants for random matrices as convolutions on the symmetric group. Probability Theory and Related Fields, $136: 19-36$.

Capitaine, M. and Donati-Martin, C. (2007). Strong asymptotic freeness for Wigner and Wishart matrices. Indiana University Mathematics Journal, 56:767-804.

Chen, J., Hontz, E., Moix, J., Welborn, M., Van Voorhis, T., Suárez, A., Movassagh, R., and Edelman, A. (2012). Error analysis of free probability approximations to the density of states of disordered systems. Physical Review Letters, 109:036403.

Chen, S., Zhang, L., and Zhong, P. (2010). Tests for high dimensional covariance matrices. Journal of the American Statistical Association, 105:810-819. 
Cima, J. A., Matheson, A. L., and Ross, W. T. (2006). The Cauchy transform. Mathematical Surveys and Monographs. American Mathematical Society, Providence, RI.

Couillet, R. and Debbah, M. (2011). Random Matrix Methods for Wireless Communications. Cambridge University Press, Cambridge, United Kingdom.

DasGupta, A. (2008). Asymptotic Theory of Statistics and Probability. Springer Texts in Statistics. Springer New York.

Dykema, K. (1993). On certain free product factors via an extended matrix model. Journal of Functional Analysis, 112(1):31-60.

Fisher, R. A. (1915). Frequency distribution of the values of the correlation coefficient in samples from an indefinitely large population. Biometrika, 10(4):507-521.

Fisher, T. J. (2012). On testing for an identity covariance matrix when the dimensionality equals or exceeds the sample size. Journal of Statistical Planning and Inference, 142(1):312-326.

Fisher, T. J., Sun, X., and Gallagher, C. M. (2010). A new test for sphericity of the covariance matrix for high dimensional data. Journal of Multivariate Analysis, 101(10):2554-2570.

Fujikoshi, Y., Ulyanov, V. V., and Shimizu, R. (2011). Multivariate Statistics: High-Dimensional and Large-Sample Approximations. John Wiley \& Sons, Hoboken.

Girko, V. L. (1990). Theory of Random Determinants. Mathematics and its applications (Soviet series). Kluwer Academic Publishers, Dordrecht, The Netherlands.

Girko, V. L. (1995). Statistical analysis of observations of increasing dimension. Kluwer Academic, New York.

Girko, V. L. and von Rosen, D. (1994). Asymptotics for the normalized spectral function of matrix quadratic form. Random Operators and Stochastic Equations, 2(2):153-161.

Glueck, D. H. and Muller, K. E. (1998). On the trace of a Wishart. Comunnications in Statistics - Theory and Methods, 27:2137-2141.

Goodman, N. R. (1963). Statistical analysis based on a certain multivariate complex Gaussian distribution (an introduction). The Annals of Mathematical Statistics, 34:152-177.

Graczyk, P., Letac, G., and Massam, H. (2003). The complex Wishart distribution and the symmetric group. The Annals of Mathematical Statistics, 31(1):287-309. 
Graczyk, P., Letac, G., and Massam, H. (2005). The hyperoctahedral group, symmetric group representations and the moments of the real Wishart distribution. Journal of Theoretical Probability, 18(1):1-42.

Greene, R. E. and Krantz, S. G. (2006). Function Theory of One Complex Variable. Graduate Studies in Mathematics Series. American Mathematical Society, Rhode Island, USA.

Gupta, A. K. and Nadarajah, S. (2004). Handbook Of Beta Distribution And Its Applications. Statistics: a Series of Textbooks and Monographs. Marcel Dekker Incorporated, New York.

Gupta, A. K. and Nagar, D. K. (1999). Matrix Variate Distributions. Monographs and Surveys in Pure and Applied Mathematics. Chapman \& Hall/CRC, Boca Raton, Florida, USA.

Haagerup, U. (1997). On Voiculescu's R- and S-Transforms for free noncommuting random variables. Free Probability Theory (Waterloo, ON, 1995), Fields Institute Communications, 12:127-148.

Haagerup, U. and Thorbjornsen, S. (2003). Random matrices with complex Gaussian entries. Expositiones Mathematicae, 21:293-337.

Hachem, W., Loubaton, P., and Najim, J. (2007). Deterministic equivalents for certain functionals of large random matrices. Annals of Applied Probability, $17(3): 875-930$.

Hanlon, P. J., Stanley, R. P., and Stembridge, J. (1992). Some combinatorial aspects of the spectral of normally distributed random matrices. Contemporary Mathematics, 138:151-174.

Harer, J. L. and Zagier, D. (1986). The Euler characteristic of the moduli space of curves. Inventiones Mathematicae, 85(3):457-485.

Hasebe, T. (2012). Fourier and Cauchy-Stieltjes transforms of power laws including stable distributions. International Journal of Mathematics, $23(3): 1250041$.

Hiai, F. (2006). Random Matrices and Free Entropy. Available October 2015 on http://www.math.uni.wroc.pl/tok/lectures/0607_Hiai_wroclaw_lect.pdf.

Hiai, F. and Petz, D. (2000). The Semicircle Law, Free Random Variables and Entropy. Mathematical surveys and monographs. American Mathematical Society, Rhode Island, USA.

James, A. T. (1964). Distributions of matrix variates and latent roots derived from normal samples. Ann. Math. Statist., 35(2):475-501.

Janik, R. A. and Nowak, M. A. (2003). Wishart and anti-Wishart random matrices. Journal of Physics A: Mathematical and General, 36:3629-3637. 
Jiang, D., Jiang, T., and Yang, F. (2012). Likelihood ratio tests for covariance matrices of high-dimensional normal distributions. Journal of Statistical Planning and Inference, 142:2241-2256.

Jiang, T. and Qi, Y. (2015). Likelihood ratio tests for high-dimensional Normal distributions. Scandinavian Journal of Statistics, (doi: 10.1111/sjos.12147).

Jiang, T. and Yang, F. (2013). Central limit theorems for classical likelihood ratio tests for high-dimensional normal distributions. The Annals of Statistics, 41:2029-2074.

John, S. (1972). The distribution of a statistic used for testing sphericity of normal distributions. Journal of Multivariate Analysis, 59:169-173.

Jonsson, D. (1982). Some limit theorems for the eigenvalues of a sample covariance matrix. Journal of Multivariate Analysis, 12:1-38.

Kawasaki, T. and Seo, T. (2012). A two sample test for mean vectors with unequal covariance matrices. Available October 2014 on http://www.math.sci.hiroshima-u.ac.jp/stat/tr/tr12/tr12-19.pdf.

Kollo, T. and von Rosen, D. (2005). Advanced Multivariate Statistics with Matrices. Mathematics and Its Applications. Springer, Dordrecht, The Netherlands.

Kollo, T., von Rosen, T., and von Rosen, D. (2011). Estimation in highdimensional analysis and multivariate linear models. Communications in Statistics - Theory and Methods, 40(7):1241-1253.

Konno, Y. (2009). Shrinkage estimators for large covariance matrices in multivariate real and complex normal distributions under an invariant quadratic loss. Journal of Multivariate Analysis, 10:2237-2253.

Ledoit, O. and Wolf, M. (2002). Same hypothesis tests for the covariance matrix when the dimension is large compared to the sample size. Annals of Statistics, 30(4):1081-1102.

Letac, G. and Massam, H. (2004). All invariant moments of the Wishart distribution. Scandinavian Journal of Statistics, 31(2):295-318.

Letac, G. and Massam, H. (2008). The noncentral Wishart as an exponential family, and its moments. Journal of Multivariate Analysis, 99(7):1393-1417.

Loh, W. L. (1991). Estimating covariance matrices. The Annals of Statistics, 19(1):283-296.

Manjunath, K. (2011). Random matrix theory : Stieltjes' transform proof (cont'd). Remarks on fourth moment assumption. Available May 2013 on http://math.iisc.ernet.in/ manju/rmt/lec 4.pdf. 
Marcinkiewicz, J. (1939). Sur une propriété de la loi de Gau $\beta$. Mathematische Zeitschrift, 44(1):612-618.

Mardia, K. V., Kent, J. T., and Bibby, J. M. (1979). Multivariate analysis. Probability and mathematical statistics. Academic Press.

Marčenko, V. A. and Pastur, L. A. (1967). Distribution of eigenvalues in certain sets of random matrices. Matematicheskii Sbornik (N.S.), 72(114):507-536.

Mathai, A. M. (1980). Moments of the trace of a noncentral Wishart matrix. Communications in Statistics - Theory and Methods, 9(8):795-801.

Matsumoto, S. (2012). General moments of the inverse real Wishart distribution and orthogonal Weingarten functions. Journal of Theoretical Probability, $25(3): 798-822$.

Mauchly, J. W. (1940). Significance test for sphericity of a normal N-variate distribution. The Annals of Mathematical Statistics, 11:204-209.

Mehta, M. L. (1991). Random Matrices. Academic Press, San Diego.

Mottelson, I. W. (2012). Introduction to non-commutative probability. Available July 2014 on

http://www.math.ku.dk/ musat/free\%20probability\%20project_final.pdf.

Muirhead, R. J. (1982). Aspects of Multivariate Statistical Theory. John Wiley \& Sons, New York.

Mukhopadhyay, P. (2009). Multivariate Statistical Analysis. World Scientific.

Müller, R. R. (2002). Lecture notes (2002-2007): Random matrix theory for wireless communications. Available June 2011 on http://www.iet.ntnu.no/ ralf/rmt.pdf.

Nagao, H. (1973). On same test criteria for covariance matrix. Annals of Statistics, 1:700-709.

Nardo, E. D. (2014). On a symbolic representation of non-central Wishart random matrices with applications. Journal of Multivariate Analysis, 125:121135.

Nel, D. G. (1971). The h-th moment of the trace of a noncentral Wishart matrix. The South African Statistical Journal, 5:41-52.

Nica, A. and Speicher, R. (2006). Lectures on the Combinatorics of Free Probability. Cambridge University Press, Cambridge, United Kingdom.

Pastur, L. A. (1972). On the spectrum of random matrices. Theoretical and Mathematical Physics, 10:67-74. 
Pielaszkiewicz, J. (2013). On the asymptotic spectral distribution of random matrices: Closed form solutions using free independence. Number 1597 in Linköping Studies in Science and Technology. Thesis. LIU-TEK-LIC-2013:31.

Rota, G.-C. and Taylor, B. D. (1994). The classical umbral calculus. SIAM Journal on Mathematical Analysis, 25(2):694-711.

Schott, J. R. (2001). Some tests for the equality of covariance matrices. Journal of Statistical Planning and Inference, 94:25-36.

Schott, J. R. (2005). Testing of complete independence in high dimensions. Biometrika, 92(4):951-956.

Schott, J. R. (2007). A test for the equality of covariance matrices when the dimension is large relative to the sampel size. Computational Statistics and Data Analysis, 51(12):6535-6542.

Seber, G. (2009). Multivariate Observations. Wiley Series in Probability and Statistics. Wiley.

Serdobolskii, V. I. (1999). Theory of essentially multivariate statistical analysis. Russian Mathematical Surveys, 54(2):351.

Silverstein, J. W. and Bai, Z. D. (1995). On the empirical distribution of eigenvalues of a class of large dimensional random matrices. Journal of Multivariate Analysis, 54(2):175-192.

Sinaı̆, I. A. G. (1991). Dynamical Systems and Statistical Mechanics: From the Seminar on Statistical Physics Held at Moscow State University by Ya. G. Sinau. Advances in Soviet mathematics. American Mathematical Society.

Speicher, R. (1994). Multiplicative functions on the lattice of noncrossing partitions and free convolution. Mathematische Annalen, 298:611-628.

Srivastava, M. S. (2005). Some tests concerning the covariance matrix in high dimensional data. Journal of the Japan Statistical Society, 35(2):251-272.

Subrahmaniam, K. (1976). Recent trends in multivariate normal distribution theory: On the zonal polynomials and other functions of matrix argument. Sankhyā: The Indian Journal of Statistics, Series A(1961-2002), 38(3):221258.

Timm, N. (2007). Applied Multivariate Analysis. Springer Texts in Statistics. Springer New York.

Tulino, A. M. and Verdu, S. (2004). Random matrix theory and wireless communications. Foundations and Trends in Communications and Information Theory, 1(1):1-182. 
Voiculescu, D. (1985). Symmetries of some reduced free product $C^{*}$-algebras. Operator algebras and their connections with topology and ergodic theory, Proc. Conf., Buşteni/Rom. 1983, Lect. Notes Math. 1132, 556-588 (1985).

Voiculescu, D. (1991). Limit laws for random matrices and free products. Inventiones mathematicae, 104(1):201-220.

Voiculescu, D. D. V., Dykema, K. J., and Nica, A. (1992). Free Random Variables. CRM Monographs. American Mathematical Society, Rhode Island, USA.

Watamori, Y. (1990). On the moments of traces of wishart and inverted wishart matrices. South African Statistical Journal, 24(2):153-176.

Wigner, E. P. (1958). On the distribution of the roots of certain symmetric matrices. Annals of Mathematics, 67(2):325-327.

Wishart, J. (1928). The generalised product moment distribution in samples from a normal multivariate population. Biometrika, 20A(1/2):pp. 32-52. 



\section{Papers}

The articles associated with this thesis have been removed for copyright reasons. For more details about these see:

http://urn.kb.se/resolve?urn=urn:nbn:se:liu:diva-122610 